\title{
Signal Power Distribution in the Azimuth, Elevation and Time Delay Domains in Urban Environments for Various Elevations of Base Station Antenna
}

\author{
Nathan Blaunstein, Martin Toeltsch, Member, IEEE, Juha Laurila, Member, IEEE, \\ Ernst Bonek, Senior Member, IEEE, Dmitry Katz, Pertti Vainikainen, Member, IEEE, \\ Nissim Tsouri, Kimmo Kalliola, and Heikki Laitinen
}

\begin{abstract}
We investigate signal power distribution in the azimuth-of-arrival, elevation-of-arrival and time-of-arrival domains for various positions of the base station antenna located below the rooftop as well as at rooftop level. This article is based on a multiparametric stochastic model we introduced in 2004, as combination of a statistical part, describing an array of buildings randomly distributed at the terrain, and a waveguide model, describing a grid of straight streets with buildings along them. Joint signal power distributions in azimuth-time delay and elevation-azimuth planes are obtained and compared to high-resolution 3-D measurements carried out in downtown Helsinki. A good agreement between theoretical predictions and the measurements is obtained basically, and also regarding the wave-guiding effect and antenna height dependencies. A satisfactory physical explanation, which accounts for the character of the specific building topography, the height and tilt of the antennas, is found. Finally, we present a numerical experiment of changing the base station antenna height, its directivity, and tilt. By this we show that the proposed stochastic approach allows to predict and control $a$-priori main parameters of smart antenna based only on knowledge of specific features of built-up terrain.
\end{abstract}

Index Terms-Azimuth, channel, comparison with measurements, delay, elevation, model, propagation, smart antennas, stochastic model, waveguide.

\section{INTRODUCTION}

D URING the last decade, an increasing demand of wireless network and multiple access performance is observed, which finally has motivated the radio engineers to develop

Manuscript received July 14, 2004; revised August 4, 2005.

N. Blaunstein and N. Tsouri are with the Department of Communication Systems Engineering, Ben Gurion University of the Negev, Beer Sheva 84105, Israel.

M. Toeltsch was with the Institut für Nachrichtentechnik und Hochfrequenztechnik, Technische Universität Wien, Vienna, A-1040 Wien, Austria. He is now with SYMENA Software and Consulting Ltd., A-1040 Vienna, Austria

J. Laurila is with the Radio Systems Area, Nokia Research Center (NRC), Radio Technologies Laboratory, Helsinki FIN-00045, Finland.

E. Bonek is with the Institut für Nachrichtentechnik und Hochfrequenztechnik, Technische Universität Wien, Vienna, A-1040 Wien, Austria.

D. Katz is with the Department of Communication Systems Engineering, Ben Gurion University of the Negev, Beer Sheva 84105, Israel and also with Albatronics Ltd., Company, Omer 84965, Israel.

P. Vainikainen is with the Radio Laboratory, Helsinki University of Technology, Espoo FIN-02015 TKK, Finland.

K. Kalliola is with the RF Measurements and Applications Group, NRC Radio Technologies Laboratory, Helsinki FIN-00045, Finland.

H. Laitinen is with the Helsinki University of Technology, Espoo FIN-02015 HUT, Finland, and also with Space Systems Finland, FIN-02200 Espoo, Finland.

Color versions of Figs. 3, 5-7, 9-11, 13-18, 20, and 22 are available online at http://ieeexplore.ieee.org.

Digital Object Identifier 10.1109/TAP.2006.882150 adaptive techniques and facilities including adaptive (also known as smart) antennas, allowing higher system capacity and more efficient spectrum utilization [1]-[14]. This process was accompanied with the significant growth of interest in developing advanced wave propagation models and for performance of appropriate high-resolution experiments, which describe temporal-spatial channel characteristics for different urban scenarios [15]-[43]. The main goal of numerous theoretical and experimental investigations was to find more applicable theoretical, deterministic, or statistical models that are able to describe most physical phenomena related to radio propagation in urban communication channels. The distribution of the total signal power at the receiver in the angle-of-arrival, azimuth (AOA) and elevation (EOA), and time-of-arrival (TOA) - usually called in the literature time delay (TD) - domains are of most interest. The users of such models are, e.g., designers of adaptive antennas, which will play a larger and larger role in modern third (3G) and forth $(4 \mathrm{G})$ generations of wireless networks [28], [43]. The high performance of adaptive antennas is based on adaptive algorithms that were derived from propagation models. It were those channel models, which enabled designers to describe the mutual AOA, EOA, and TD distributions of the total signal intensity, and which at the same time could describe different situations in the urban scene covering various parameters such as building density, building overlay profile, and street orientation with respect to the terminal antennas together with their height, directivity, and pattern, which play an significant role in signal power distribution within the area of service.

Most experimental investigations of temporal and spatial variations of radio waves carried out in recent decades for various conditions of terrain showed that a wide spectrum of shadow zones is observed at the street level, and very sharp boundaries between light and shadow zones are created [25]-[28]. Moreover, it was found that various urban scenarios with specific peculiarities of the topography influence in different manners on the mutual AOA, EOA, and TD distributions of the total signal [20]-[22], [31]-[38], [43]. From the point of view of designing multiple access networks, it is very important to use some unified spatio-temporal approach for various situations in the urban scene.

Unfortunately, most existing propagation models include only a partial description of the real urban channel in terms of AOA, TD or mutual AOA-TD distributions. The reason is that currently there is no general algorithm to predict signal 
power characteristics in space, time, and frequency domains for urban and suburban environments. Each specific model describes a special scenario in the urban scene and requires only statistical description of the real building pattern inside the city, instead of using well-known deterministic approaches like the field characteristics inside the propagation channel. The main disadvantage of these specific models-statistical [16], [17], [29]-[31], measurement-based [32]-[34], or ray-tracing [35], [36]—is the absence of the urban area dependent unified parameters of the model that adapt the results of measurements to the type of the corresponding environment.

Obviously, an accurate prediction or performance analysis of the adaptive system, based on these methods, requires a lot of measurements to be done with different types of calibration techniques for different types of environments in each investigated area. First this was done in [18]-[22], as an approach that introduces urban propagation mechanism based on 3-D high-resolution radio channel measurements carried out in different European cities, particularly in Helsinki (Finland) and Paris (France). This approach has analyzed the propagation mechanism using comprehensive classification of different types of wave propagation in urban scene. The classification defines three propagation classes: street-guided propagation (class 1), direct propagation over the rooftop (class 2), and scattering reflection from high-rise objects (class 3). We will compare our proposed stochastic approach with measurements carried out in Helsinki [21], [22]. As can be summarized from results obtained in those publications, the detailed understanding of radio propagation phenomena is a key concept for the development and performance assessment of mobile and personal communication networks.

This paper continues to analyze a new multiparametric stochastic approach, proposed earlier in [42], [43] for the separate AOA and TD signal distribution, and also for their joint distribution [44], in urban environments for various heights of base station antennas where it was shown, through numerous experiments [31]-[34], that this model is applicable and effective in understanding joint AOA and TD effects in various urban environments. In Section II, we briefly introduce our stochastic model and then modify it, taking into account the guiding effects caused by the broken - by crossing streets-waveguides (mutlislit) [27], [40], [41], as an additional phenomenon in the existing stochastic propagation channel model. An antenna pattern model in the EOA domain is briefly investigated here to describe the influence of the vertical antenna pattern on the AOA and TOA signal power distributions. Section III presents simulation results. Here, the comparison with experimental data is performed, based on experiments published in [21], [22].

\section{A General 3-D Stochastic Model}

This section describes how disadvantages of current models can be eliminated by advanced techniques. In Section II-A, the concept of a multiparametric stochastic approach is introduced briefly based on results obtained in [42], [43]. The main distinction of the proposed model is the introduction of a statistical description of the urban propagation environment. Section II-B describes the street waveguide effects examined in [27], [40], [41] and then, in Section II-C, a novel consideration of the guiding effect caused by the broken street waveguide (multislit) in the urban channel and the angle-of-arrival (AOA) and time-delay (TD) signal power distributions is presented, following [44]. Section II-D is dedicated to the effect of directional antennas with various tilts at the base station and the effect on the elevation-of-arrival (EOA) signal distribution.

\section{A. Azimuth-of-Arrival and Time Delay Signal Power Distributions}

To describe fully the urban terrain and the antenna heights the following parameters have been introduced in [27], [42], [43] according to the proposed stochastic model.

The average horizontal distance of LOS, $\gamma_{0}^{-1}$, defined by

$$
\gamma_{0}^{-1}=\langle\rho\rangle=\frac{\pi}{2\langle L\rangle \nu}
$$

the minimum and maximum building height in the area of investigation, $h_{1}$ and $h_{2}$, and the corresponding terminal antenna heights, for mobile station $z_{1}$ and for base station $z_{2}$ where $z_{2}>z_{1}$.

In (2.1) $\langle L\rangle$ is the average length of buildings, $\nu$ is the density of buildings per square kilometer.

Following [27], [42], and [43], let us now present the function of spatial variations of the total field in the urban propagation channel with randomly distributed obstacles. As in previous works concerning the multiparametric stochastic model, we put the origin of the coordinate system $\{x, y, z\}$ at the point $B\left(\mathbf{r}_{2}\right)$, which now describes the moving receiver (see Fig. 1). At the moment $t>0$, the receiver passes the point $D\left(\mathbf{r}_{2}\right)$ with velocity $\mathbf{v}$ in a direction that is determined by the angle $\varphi_{0}$. The base station, as the transmitter, is placed at the point $A\left(\mathbf{r}_{1}\right)$ with its coordinates $\left(d, 0, z_{1}\right)$; the point $\mathrm{C}\left(\mathbf{r}_{\mathrm{s}}\right)$ is the point of scattering for the arbitrary ray ABC. As is shown in Fig. 1, during the time $t$ the mobile receiver will arrive at point $D\left(\mathbf{r}_{2}^{\prime}\right)$ from point $B\left(\mathbf{r}_{2}\right)$. Using results obtained in [42], [43], we can present the function of field correlation $K\left(\mathbf{r}_{2}, \mathbf{r}_{2}^{\prime}\right)=\left\langle U\left(\mathbf{r}_{2}\right) U *\left(\mathbf{r}_{2}^{\prime}\right)\right\rangle$ as

$$
\begin{aligned}
K\left(\mathrm{r}_{2}, \mathrm{r}_{2}^{\prime}\right) \approx & K\left(\lambda, \varphi_{0}\right) \\
= & \int_{1}^{\infty} d \tau \int_{0}^{2 \pi} d \varphi \frac{\left\langle\sigma\left(r_{2}, r_{s}, r_{1}\right)\right\rangle}{r^{2} \widetilde{r}^{2}} P(l, \tau, \varphi) \mu(\tau, \varphi) \\
& \times \exp \left\{i k l \cos \left(\varphi-\varphi_{0}\right)\right\} .
\end{aligned}
$$

Here, we introduce a new variable of integration $\tau=$ $(r+\widetilde{r}) / d$; where $\tau \in(1, \infty)$, the azimuth $\varphi$ in reference to the axis $\mathrm{AB}$ (see Fig. 1), and a new variable $\widetilde{r}=\sqrt{\left(x_{2}-x\right)^{2}+\left(y_{2}-y\right)^{2}}=\left(d^{2}+r^{2}-2 r d \cos \varphi\right)^{1 / 2}$. The function $\mu(\tau, \varphi)$ is the density of the joint distribution (of both the time delay $\tau$ and the azimuth $\varphi$ ) of the reflected waves at the receiver obtained in [42], [43]. Function $P(l, \tau, \varphi)$ is the probability that it is not a shadowing effect for the segment $l$ at the screen-scatterer, which can be presented in coordinates $(\tau, \varphi)$ as

$$
P(l, \tau, \varphi)=\exp \left[-\frac{\pi \gamma_{0} d}{4} \frac{l}{L} \frac{\left(\tau^{2}-1\right)}{(\tau-\cos \varphi)}\left|\sin \left(\varphi-\varphi_{0}\right)\right|\right] .
$$




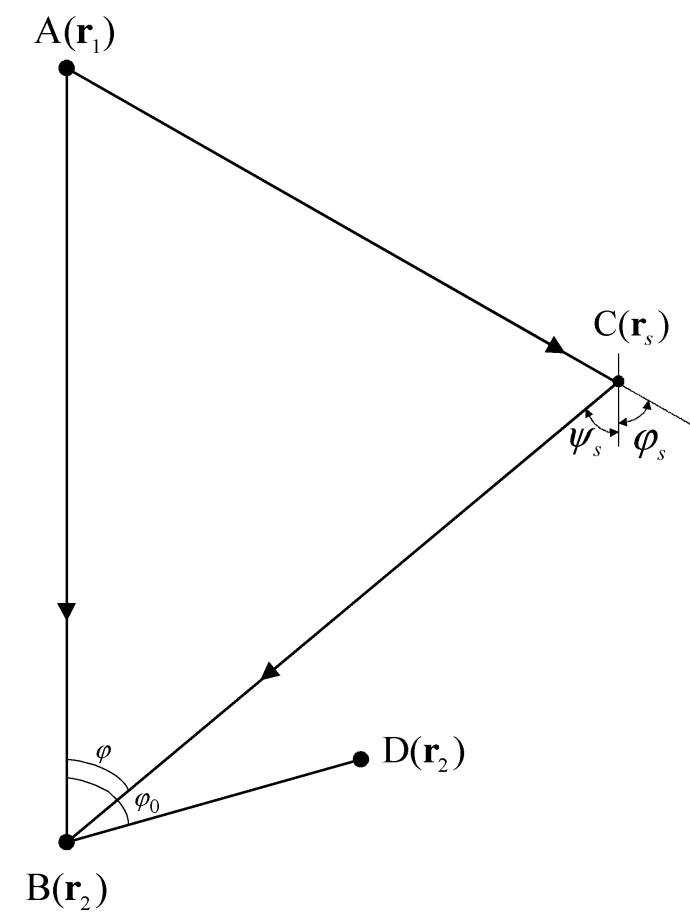

Fig. 1. The geometry of scattering from a nontransparent building.

As was shown in [42], [43], the function $\mu(\tau, \varphi)$ has a sharp maximum in the region close to the receiver and transmitter locations. At the same time the probability $P(l, \tau, \varphi)$, described by (2.3), is a smooth function. For the case of $\left|\sin \varphi_{0}\right|>\left(\gamma_{0} d\right)^{-1}$, i.e., when points B and D are not so far from each other along the radio path (see Fig. 1), we can integrate (2.2) over the variable $\tau$ using the well-known Laplace method [42], [43] and finally obtain for $\gamma_{0} d \gg 1$

$$
\begin{aligned}
& K\left(l, \varphi_{0}\right)=\exp \left\{-\frac{\nu L}{2}\left|\sin \varphi_{0}\right|\right\} \\
& \times \int_{0}^{2 \pi} W(\varphi) \exp \left\{i k l \cos \left(\varphi-\varphi_{0}\right)\right\} \frac{d}{2 \pi} .
\end{aligned}
$$

Here the function $W(\varphi)$ determines the energy spectrum of the total field scattered from any scatterer for any observer placed at the mobile receiving point $\mathrm{B}$, i.e., the signal power distribution over the azimuth. It can be obtained for the case of $\gamma_{0} d \gg 1$ taking into account diffraction from the virtual sources and the method of integration of (2.2) described in [42], [43]

$$
W(\varphi)=\frac{\Gamma \lambda l_{\nu} h}{16 \pi^{2}\left[\lambda^{2}+\left(2 \pi l_{\nu} \gamma_{0} \bar{h}\right)^{2}\right] d^{3}}\left\{f_{1}(\varphi)+f_{2}(\varphi)\right\}
$$

where

$$
\begin{aligned}
f_{1}(\varphi)= & \frac{2 z_{1}^{2}\left(\gamma_{0} d\right)^{2}}{\left(z_{2}+\bar{h}\right)} \frac{\varsigma^{\prime}(1-\cos \varphi)}{\bar{h}} \\
& \times \frac{\exp \left[-\gamma_{0} d\left(\frac{\bar{h}}{z_{2}}+\frac{\varsigma^{\prime}}{2} \frac{(1+\cos \varphi)}{\left[1+\frac{\gamma_{0} d}{2}\left(1+\frac{\bar{h}}{z_{2}}\right)(1-\cos \varphi)\right]}\right)\right]}{\left[1+\frac{\gamma_{0} d}{2}\left(1+\frac{\bar{h}}{z_{2}}\right)(1-\cos \varphi)\right]}
\end{aligned}
$$

$$
\begin{aligned}
f_{2}(\varphi)= & \frac{2 h\left(\gamma_{0} d\right)}{\left(z_{2}+\bar{h}\right)}\left[1+\left(\frac{\bar{h}}{z_{2}}\right) \frac{1+\left(k \lambda_{\nu} \gamma_{0} d\right)^{2}}{1+\left(\gamma_{0} \varsigma^{\prime} d\right)^{2}}\right] \\
& \times \frac{\exp \left[-\gamma_{0} d\left(\frac{\bar{h}}{z_{2}}+\frac{\varsigma^{\prime}}{2} \frac{(1+\cos \varphi)}{\left[1+\frac{\gamma_{0} d}{2}\left(1+\frac{\bar{h}}{z_{2}}\right)(1-\cos \varphi)\right]}\right)\right]}{\left[1+\frac{\gamma_{0} d}{2}\left(1+\frac{\bar{h}}{z_{2}}\right)(1-\cos \varphi)\right]} .
\end{aligned}
$$

Here, $\Gamma$ is the absolute value of the reflection coefficient from the building surface; $l_{\nu}$ is the height or width of building's segments, $k=2 \pi / \lambda, l$ is the a wavelength, $z_{1}$ is the height of the MS (denoted in Fig. 2 as $H_{R}$ ), $z_{2}$ is the height of the BS (denoted in Fig. 2 as $H_{T}$ ), $\varphi$ is the angle-of-arrival (i.e., azimuth), $\bar{h}$ is an average height of the building, and $d$ is distance between receiver and transmitter. Here also a new parameter

$$
\varsigma^{\prime}=\frac{\left[\left(\lambda d / 4 \pi^{3}\right)+\left(z_{2}-\bar{h}\right)^{2}\right]^{\frac{1}{2}}}{z_{2}}
$$

which accounts for the process of diffraction over the buildings, was introduced according to [42], [43] instead of $\varsigma=$ $\left(z_{2}-\bar{h}\right) / z_{2}$ used in [25] where diffraction phenomena were not considered. The factor $\left(\lambda d / 4 \pi^{3}\right)$ in (2.7) describes the effect of the "knife-edge" diffraction from roofs and the degree of clearance of the first Fresnel zone with radius $\sim \sqrt{\lambda d}$. As was shown in [42], without accounting effects of diffraction, i.e., with absence of the term $\left(\lambda d / 4 \pi^{3}\right)$ in (2.6), we get no correct physical solutions for $W(\varphi)$ for the case when $z_{2} \leq \bar{h}$ cf. [25].

There are two main terms $f_{1}$ and $f_{2}$ in the (2.5). Each of them relates to a different propagation phenomenon. The term $f_{1}$ is the significant term that describes the influence of the scattering area located in the proximity of the MS. The term $f_{2}$ describes general effects of other scatterers that distribute uniformly in areas surrounding the BS and MS. The influence of different scatterers for the typical three cases, depending on the BS antenna height, is sketched in Fig. 2.

When the height of the antennas is below the building profile height [Fig. 2(c)], both components $f_{2}$ and $f_{1}$ are significant for the distribution $W(\varphi)$. From (2.3) follows that, if the base station antenna height increases up to $z_{2}=\bar{h}$ [Fig. 2(b)], then $\varsigma^{\prime}=$ $\sqrt{\left(\lambda d / 4 \pi^{3}\right)} / z_{2} \ll 1$ and $f_{1}(\varphi) \rightarrow 0, f_{2}(\varphi)_{z_{2}=h}>f_{1}(\varphi)_{z_{2}=h}$ In this case of $z_{2}=\bar{h}, f_{1}$ is close to zero, and that means that all scatterers located in the far zone from MS, near the BS, will influence the spreading of the total signal at the BS; we can relate to the disk region of scatterers presented by Fig. 2(b). With increasing height of the $\mathrm{BS}$ antenna, i.e., $z_{2}>h$, the influence of buildings surrounding the MS on the total signal distribution will be more significant, and $f_{1}$ becomes larger than $f_{2}$, describing the effect of scatterers located close to MS [Fig. 2(a)]. When the BS antenna is above the rooftop level [Fig. 2(a)], the spectrum distribution in azimuth domain $W(\varphi)$ depends only on the position and distribution of scatters (obstructions) close to the MS; they are uniformly distributed in a circle (disk) close to the MS [Fig. 2(a)].

In this case, the influence of scatterers (buildings) in the proximity of the MS on the signal received at the MS is increased and contributes more than scatterers surrounding the BS [Fig. (1)] and also those located far from the MS. 

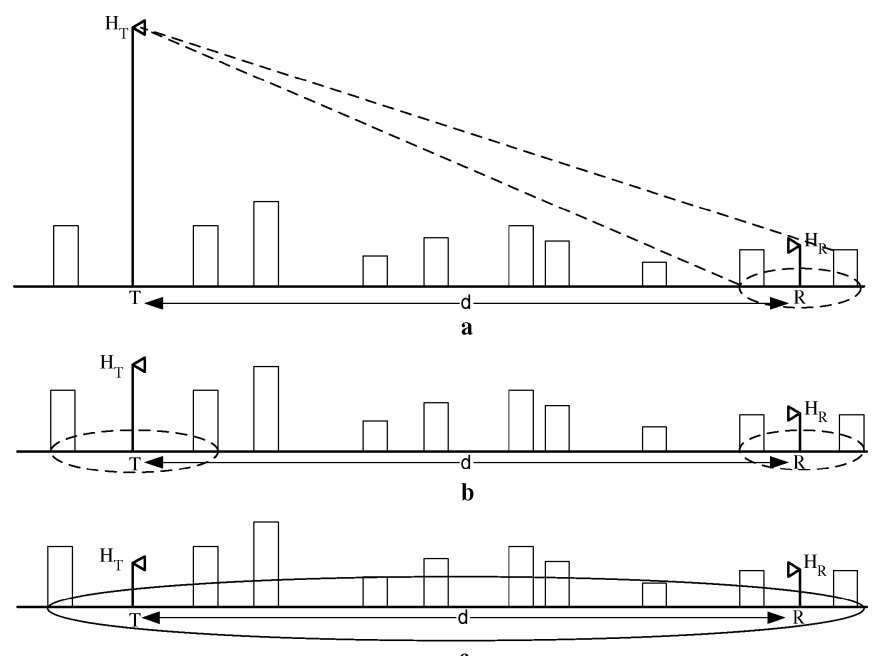

Fig. 2. Geometry of scatterers depending on antennas heights. (a) BS height larger than buildings, (b) BS on rooftop level, (c) BS below buildings.

In a similar way, the power spectrum of time delay of arriving waves was obtained in [42], [43]

$$
\begin{aligned}
W(\tau)= & \frac{\Gamma}{8 \pi^{2} d^{2}} \frac{k l_{\nu} \gamma_{0} \bar{h}}{1+\left(k l_{\nu} \gamma_{0} \bar{h}\right)^{2}} \\
& \times\left\{\left(1-\varsigma^{\prime}\right)\left[1+\left(1-\varsigma^{\prime}\right)^{2} \frac{1+\left(k l_{\nu} \gamma_{0} \bar{h}\right)^{2}}{1+\left(\varsigma^{\prime} \gamma_{0} \bar{h}\right)^{2}}\right] f_{1}(\tau)\right. \\
& \left.\quad+\frac{\varsigma^{\prime}}{\left(1-\varsigma^{\prime}\right)} f_{2}(\tau)\right\}
\end{aligned}
$$

where

$$
\begin{aligned}
f_{1}(\tau)= & \frac{\left(\gamma_{0} d\right)^{2} \sqrt{\tau^{2}-1}}{4 \tau^{2}} \\
& \times \exp \left\{-\gamma_{0} \tau \frac{\left(2-\varsigma^{\prime}\right)}{2} d\right\} I_{0}\left(\frac{\gamma_{0} \varsigma^{\prime} d}{2}\right) \\
f_{2}(\tau)= & \frac{\gamma_{0} d}{2} \exp \left\{-\frac{\gamma_{0} \tau d}{2}\right\} \\
& \times\left[\exp \left\{-\frac{\gamma_{0} \tau d}{2}\right\}+\frac{\sqrt{\tau-1}}{\sqrt{\tau+1}} I_{0}\left(\frac{\gamma_{0} d}{2}\right)\right] .
\end{aligned}
$$

Here we introduced, relationships between $\widetilde{r}, r, d$, and relative time $\tau$ of signal arrival with respect to that of pseudo-LOS component, which is defined as the wave-component passing along the virtual axis between the transmitter and the receiver: the variable $\tau$ is the time delay

$$
\widetilde{r}(\tau, \varphi)=\frac{d\left(\tau^{2}-2 \tau \cos \varphi+1\right)}{2(\tau-\cos \varphi)}, \quad r(\tau, \varphi)=\frac{d\left(\tau^{2}-1\right)}{2(\tau-\cos \varphi)}
$$

and $\varphi$, is azimuth.

In the time delay domain, the same properties of the signal power spectrum, as above for the azimuth domain, can be obtained. Thus, in the case of $z_{2} \leq \bar{h}$

$$
\frac{\varsigma^{\prime}}{\left(1-\varsigma^{\prime}\right)} f_{2}(\tau) \gg\left[1+\frac{1+\left(k l_{\nu} \gamma_{0} \bar{h}\right)^{2}}{1+\left(\varsigma^{\prime} \gamma_{0} \bar{h}\right)^{2}}\right] f_{1}(\tau)
$$

and the distribution of scatterers far from BS antenna, and close to MS antenna [see Fig. 2(a)] does not influence on a power spectrum $W(\tau)$ in the time delay domain. Then, with increasing height of the receiver antenna (when $z_{2}>\bar{h}, \varsigma^{\prime}>1$ ) the main contribution follows from the first term with $f_{1}(\tau)$, which describes the influence of the neighboring area around the MS. In this case, condition (2.11) must be converted. We also note that the function $f_{2}(\tau)$ does not depend on parameter $\varsigma^{\prime}$, i.e., on receiving antenna's height with respect to the rooftops. Therefore, for $\varsigma^{\prime}>0.3$ the form of the power spectrum versus time delay becomes almost constant.

\section{B. Street Guiding Effect in Urban Areas}

Set of experiments in urban areas with randomly distributed buildings placed on rough terrain were done in [20]-[22]. A guiding effect was observed (cf., Section III) during these experiments as significant propagation phenomenon in urban areas, particularly when both terminal antennas are placed below or at the rooftop level.

The concept in the current paper is based on the idea that EM-waves propagate along a street in a city area as if the street was structured in the form of a broken (multislit) waveguide [27], [40], [41]. The proposed model considers the multiple reflections from building walls, multiple diffractions by walls' corners and reflection from the road surface. The street is considered as a broken waveguide with Poisson distribution of buildings and intervals between them (slits) according to [27], [40]

$$
\begin{gathered}
f\left(L_{n}\right) \sim \frac{1}{\langle L\rangle} \exp \left\{-\frac{L_{n}}{\langle L\rangle}\right\} \\
f\left(l_{n}\right) \sim \frac{1}{\langle l\rangle} \exp \left\{-\frac{l_{n}}{\langle l\rangle}\right\}
\end{gathered}
$$

where $L_{n},\langle L\rangle$ and $l_{n},\langle l\rangle$ are screen and slit lengths and their mean values, respectively. Each screen (building) is described by its electrical properties, defined by a surface impedance and finally by the absolute value of its reflection coefficient. Let us define a discrete spectrum of total field intensity within a broken waveguide according to [27], [40]

$$
W(r)=\frac{C^{2}}{r} \exp \left[-2 \frac{|\ln \chi \cdot \Gamma|}{\rho_{n}^{0} a}\left(\frac{\pi n-\varphi_{n}}{a}\right) r\right]
$$

where $C$ is a constant defined by relation between antenna parameters [27], [40], $r$ is the BS-MS distance, $\chi$ is a brokenness parameter defined by

$$
\chi=\frac{\langle L\rangle}{\langle L\rangle+\langle l\rangle}
$$

$a$ is the width of the street, $\rho_{n}^{0}$ is the wave number defined by

$$
\rho_{n}^{0}=\sqrt{k^{2}+\left(\frac{n \pi}{a}\right)^{2}}
$$

$\Gamma$ is the absolute value of the reflection coefficient, $\varphi_{n}$ is the phase of the reflection coefficient, $n$ is the number of reflections (wave modes), and $k=2 \pi / \lambda$. Without loss of generality, we assume that the buildings' walls are close to perfectly conductive 


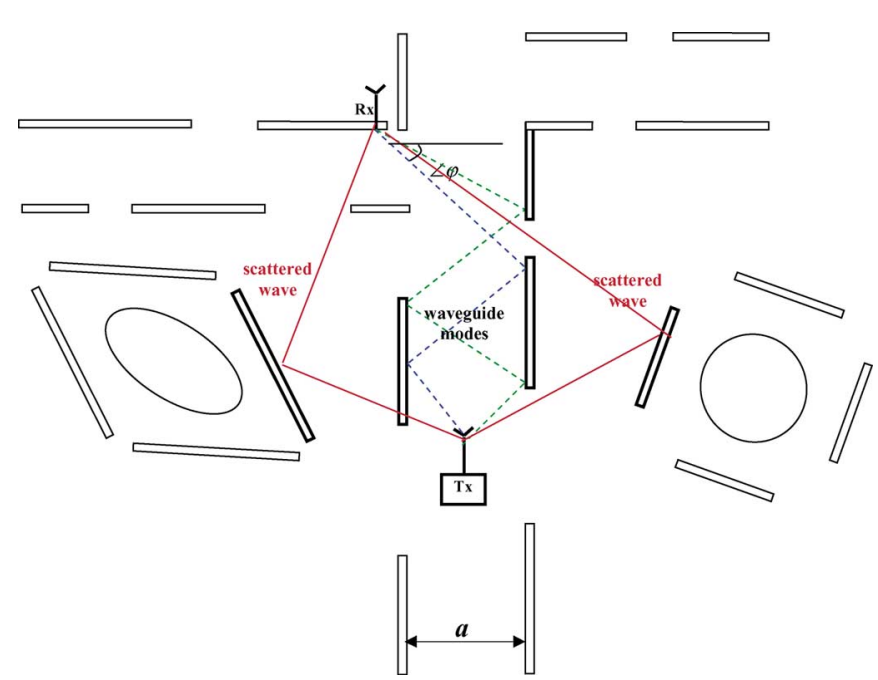

Fig. 3. 2-D model of a street waveguide accompanied by scatters randomly distributed around the transmitter and receiver.

walls, and for this case $\Gamma=1$ and $\varphi_{n}=180^{\prime}$. Such assumptions allow us to rewrite (2.13) in the following form:

$$
W(r)=\frac{C^{2}}{r} \exp \left[-2 \frac{|\ln \chi|}{a^{\prime}} \cdot r\right]
$$

where $a^{\prime}=\sqrt{\left(4 a^{4} / \lambda^{2} n^{2}\right)+a^{2}}$. In our future computations we assume the wave number $n$ to be unity, because, as was proved experimentally in [27], [40], [41], the wave modes with $n>1$ attenuate very fast along the street waveguide.

We will rewrite now (2.16) by introducing a polar coordinate system, as it is shown in Fig. 3. According to the geometry presented in Fig. 3, using the relations between variables (2.10), we can rewrite formula (2.16), following [44], as

$W_{w g}(\tau, \varphi)=\mathrm{W}_{0} \frac{2(\tau-\cos \varphi)}{d\left(\tau^{2}-1\right)} \exp \left[-2 \frac{|\ln \chi|}{a^{\prime}(\varphi)} \cdot \frac{d\left(\tau^{2}-1\right)}{2(\tau-\cos \varphi)}\right]$

where $W_{0}$ is a signal power of the isotropic antenna in free space. Now, to find the total signal power distribution in time and azimuth domains, accounting the guiding street effect, we combine, formula (2.17) with (2.5)

$$
\mathrm{W}_{\mathrm{fin}}(\varphi)=\mathrm{W}(\varphi) \cdot \mathrm{W}_{\mathrm{wg}}(\varphi)
$$

or with $(2.8)$

$$
\mathrm{W}_{\mathrm{fin}}(\tau)=\mathrm{W}(\tau) \cdot \mathrm{W}_{\mathrm{wg}}(\tau)
$$

to determine the joint 2-D distribution in the azimuth-of-arrival (AOA) and time-delay (TD) domains, respectively. In above formulas $\mathrm{W}_{\mathrm{wg}}(\varphi)$ can be obtained by integration of (2.17) over $\tau$, and $\mathrm{W}_{\mathrm{wg}}(\tau)$ can be obtained by integration of (2.17) over $\varphi$ [42], [43].

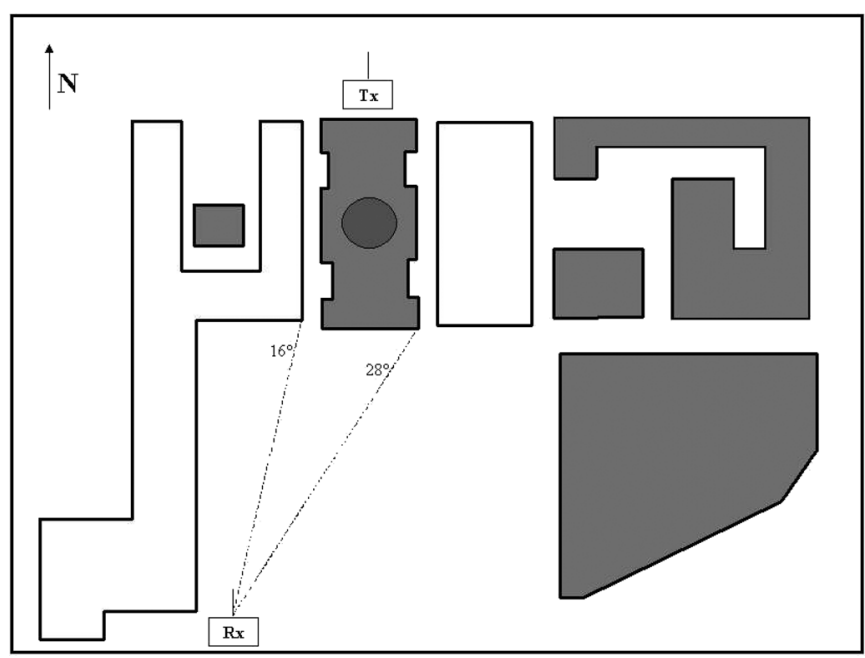

Fig. 4. Micro-cell urban environment ( $\mathrm{Rx}$ is below the rooftop level).

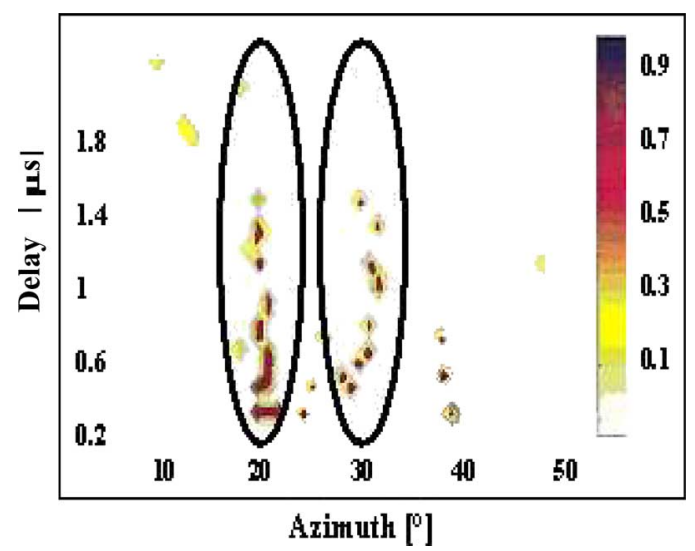

Fig. 5 Measured data in AOA-TOA plane for antenna below the rooftop level.

\section{Effects of Antenna Directivity in the Elevation Angle Domain}

Considering adaptive antennas, we will use a simplified engineering approach based on two projections, horizontal and vertical, to depict the antenna 3-D pattern, which defines the antenna gain as a function of azimuth and elevation angles, respectively. For this purpose we use the von Mises probability density function (PDF), which fully satisfies our requirements to describe a behavior of the directional antenna. This distribution was investigated in details applying to the statistical modeling and analysis of angular variables [24], [39], and is very effectively used in radar applications. We investigate both antenna directivity and tilt effects assuming permanent omnidirectional antenna pattern in the azimuth domain and variable antenna pattern, according to von Mises distribution, in elevation domain.

Let the variable $\theta$ represent the elevation angle and $\beta$ represent the antenna tilt ( $\beta>0$ denotes above the horizon, $\beta<0$ denotes below the horizon). The von Mises distribution is given by

$$
\mu(\theta)=\frac{1}{2 \pi I_{0}(\kappa)} \exp [\kappa \cos (\theta-\beta)]
$$



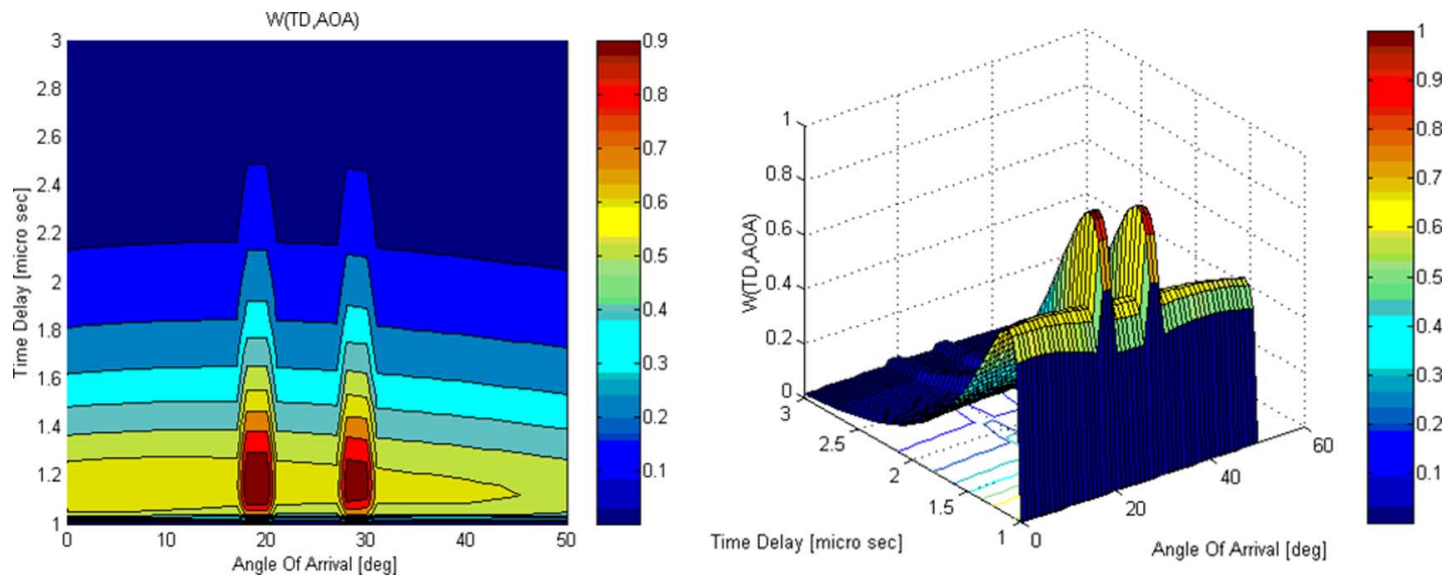

Fig. 6. Joint AOA-TD relative signal power distribution in (a) 2-D and (b) 3-D pictures.

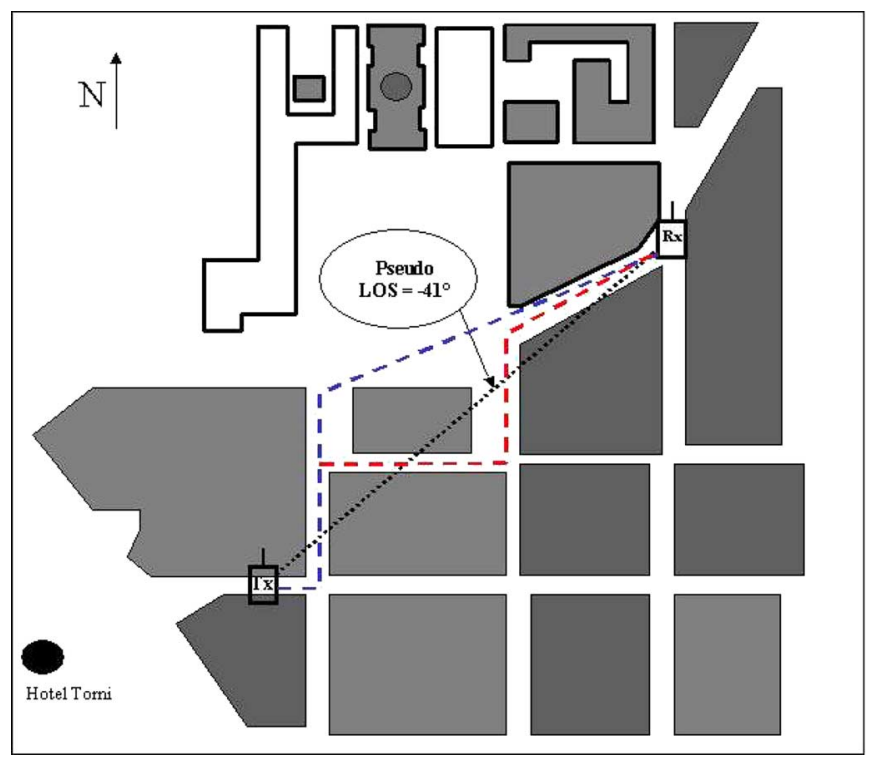

Fig. 7 Micro-cell urban environment (RX is at the rooftop level).

where $I_{0}($.$) is the zero-order modified Bessel function, and \kappa$ is an antenna directivity parameter [39]. We will analyze this formula for different parameters $\theta$ and $\beta$.

The corresponding signal intensity distribution in the EOA domain can be easily obtained taking into account the same derivation as was done for AOA and TOA domains, accounting their mutual independency. Following the same procedure of derivation mentioned in [42]-[44], we get

$$
W(\theta)=\frac{W_{0}}{2 \pi I_{0}(\kappa)} \exp [\kappa \cos (\theta-\beta)]
$$

where, $W_{0}$ is the signal power of the isotropic antenna in LOS conditions. Formula (2.21), combining it with (2.18) or with (2.19), describes the joint signal power spectrum in EOA-AOA and EOA-TD planes, respectively.

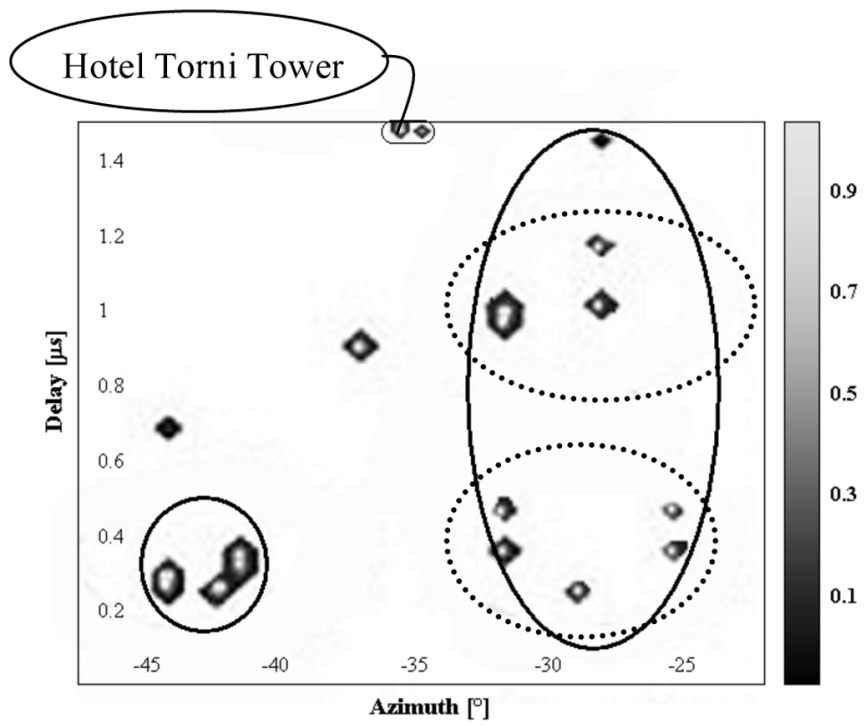

Fig. 8. Measured data in the AOA-TD plane: antenna is at the rooftop level.

\section{NumericAl SimUlation Versus Results OF MEASUREMENTS}

In the previous section we introduced and analyzed the multiparametric and multislit street waveguide models. Below we compare numerical simulations of the mutual AOA, EOA and TD distributions with the measurement results presented in [21], [22]. Let us first of all briefly describe the 3-D high-resolution measurements of the spatial radio channel carried out in different scenarios of downtown Helsinki.

\section{A. TD-AOA Plane: Comparison of Theory and Experiment}

As was mentioned above, according to the geometry of scatterers, the buildings' overlay profile compared with the terminal antenna heights (see Fig. 2), all types of structures between the receiver and the transmitter antennas, play a significant role in microcell radio environment. Simply speaking, each urban area has its own fingerprint of AOA, EOA, and TD. Let us now prove those statements thorough the prism of the corresponding 3-D 

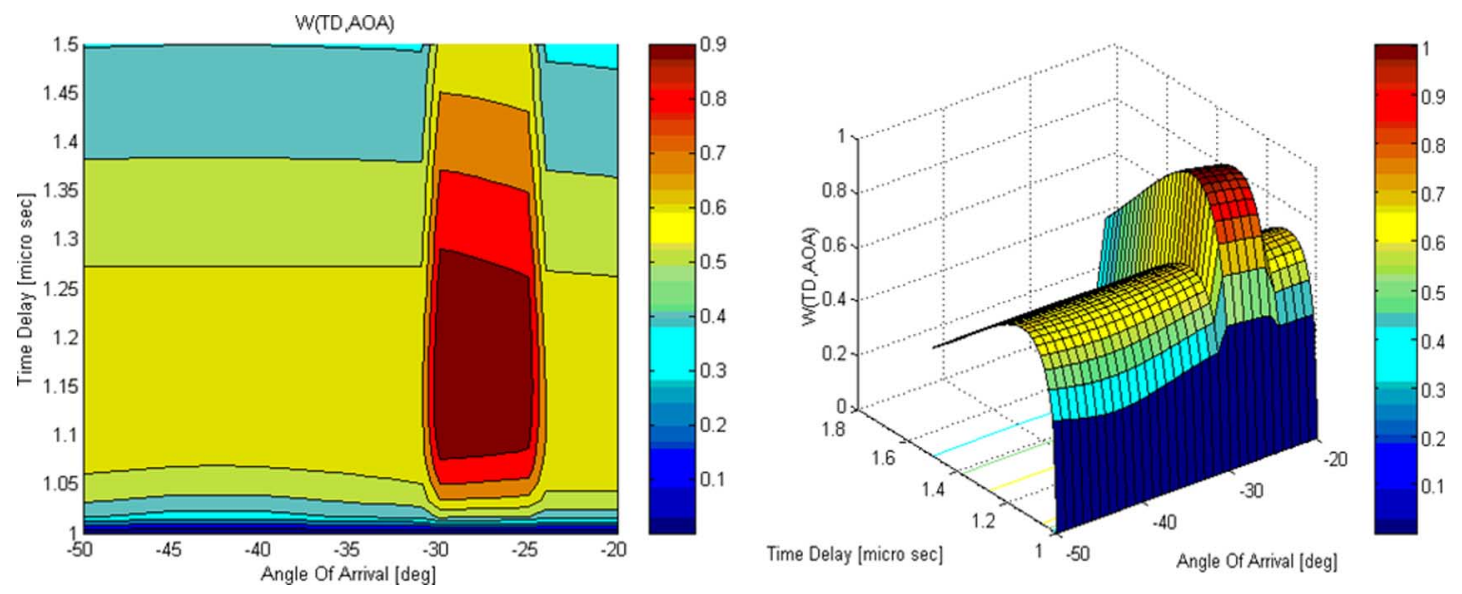

Fig. 9. Joint AOA-TD relative signal power distribution in (a) 2-D and (b) 3-D pictures.

high resolution measurements described in [21], [22], where different receiver sites with different positions were investigated.

1) Antenna Location Below the Rooftops: The first site denoted by RX1 is characterized by the antenna located on the third floor of a building below the rooftops at the height of $h_{R X}=10 \mathrm{~m}$ (see Fig. 4, taken from experiments in [21], [22]).

The experiment above has been done in the following conditions: minimum traffic on the streets, TX antenna kit is on street level $+2 \mathrm{~m}$, its pattern is omnidirectional in the azimuth and has $87^{\circ}$ beamwidth in the elevation $3 \mathrm{~dB}$ beamwidth of RX antenna is $70^{\circ}$ both for azimuth and elevation, which correspond to $\kappa=5$. A wide-band ( $100 \mathrm{MHz})$ channel sounder has been used with a carrier frequency of $2.154 \mathrm{GHz}$. The average height of buildings located at the RX-site is $\bar{h} \approx 20-21 \mathrm{~m}$.

Let us now examine in Fig. 5 the resulting image of the joint AOA and TD distribution of the signal power received by post-processing of the measured data at [21], [22]. We show the corresponding simulations of the relative signal power joint TD-AOA distribution, $\widetilde{W}(\tau, \varphi)=\mathrm{W}(\tau, \varphi) / W(0,0)$, as combination of (2.5), (2.8) and (2.17), in Fig. 6 in the (a) 2-D plane and (b) 3-D plane. Here $W(0,0)$ is the total signal power in the pseudo-LOS direction, as the virtual direct radio path between the transmitter and the receiver. The parameters, which describe the urban scene presented in Fig. 4, are $\gamma_{0}=4 \mathrm{~km}^{-1}\left(\rho=\gamma_{0}^{-1}=250 \mathrm{~m}\right)$, the average value of the reflection coefficient $\Gamma=0.7$, the parameter of brokenness $\chi=0.5$, the TX-RX distance $d=0.3 \mathrm{~km}$, and the wavelength $\lambda=0.13 \mathrm{~m}$. As follows from measurement results presented in Fig. 5, and was predicted in [21], [22], more than 80-90\% of the power of rays arrive at the receiver from two streets due to guiding effects, i.e., by multiple reflections from buildings located along the streets. Those measured results completely support our results of the simulations presented in Fig. 6 for the experimental site shown in Fig. 4.

Thus, the maximum of energy arrives at the receiver from the directions to the two parallel streets, i.e., from 20 to 30 degrees of azimuth. The maximum energy arrives at the receiver with time delay of 1-1.5 $\mu \mathrm{s}$, whereas the measured time delay of main rays arriving through these two streets varies between 0.5 and $1 \mu \mathrm{s}$, twice as small as obtained in simulations. This difference between computed and measured values can be explained

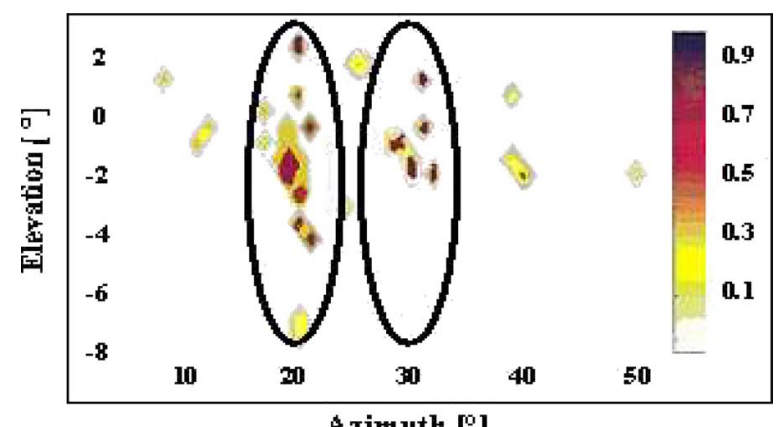

Fig. 10. Measured data in the EOA-AOA plane: antenna is below the rooftop level.

by the fact that a LOS distance between Rx and Tx of $300 \mathrm{~m}$ was taken into account as a reference, giving a minimum time delay of $1 \mu \mathrm{s}$. However, as was shown also in [23], the average measured time delay for the experimental site shown in Fig. 4 can achieve 2-3 microseconds. So, we can point out that the data predicted both theoretically and experimentally are completely verifiable. Furthermore, the guiding effect of two streets shown in Fig. 4 is clearly seen both from measured data and results of computations.

All figures, obtained experimentally and theoretically, show that besides the fact that the dominant energy of the total signal (more than 80\%) is caused by the guiding effect of streets, there is a portion of total multipath energy (about 15-20\%), arriving from different directions with symmetry around the pseudo-LOS direction. In addition, the maximum arriving energy has minimum time delay in the direction close to the radio path through the street canyons. This result is clearly understood by means of the analysis of (2.17)-(2.19) for the case of TX and RX antennas below the rooftop level. Therefore, we can summarize that the number of scatterers, influencing on the arriving multipath energy from TX direction, exponentially vanishes with increase of azimuth and time delay. This result was obtained experimentally in [21], [22] and was explained based on phenomenological vision of the propagation mechanism. The new principal result is the consideration of the real street orientation provided by our novel stochastic multiparametric model with waveguide effect extension. 


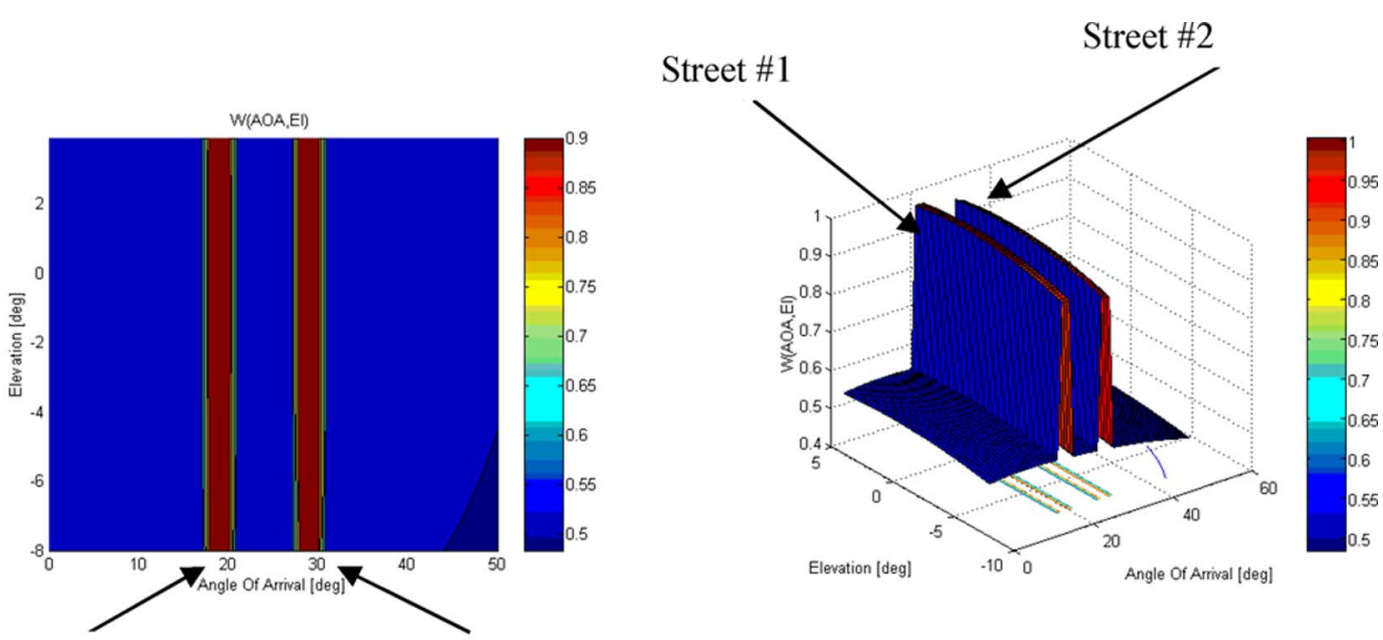

Street\#1

Street\#2

Fig. 11. Joint EOA-AOA relative signal power distribution in (a) 2-D and (b) 3-D pictures.

2) Receiving Antenna at the Rooftop Level: Let us now investigate another set of measurements, which is related to the case, denoted as RX2 in [21], [22], where the RX antenna was at the same level as the rooftops $\left(h_{R X}=27 \mathrm{~m}\right)$. The experiment, shown in Fig. 7, has been done in the following conditions: there is no traffic on the streets, the TX and RX antenna properties were the same as in Section III-A.1. Again, there is no LOS between the TX and RX antenna. The distance between the terminals was about $420 \mathrm{~m}$. One wide street is placed with azimuth of about $-30^{\circ}$ from the main lobe of the RX antenna ( $\mathrm{RX}$ is mounted at $270^{\circ}$ from the North direction).

Fig. 8 shows the result image of the post-processed measured data for the area shown in Fig. 7. Fig. 9(a) and (b) shows the result image of simulating the relative signal power $\widetilde{W}(\tau, \varphi)=$ $\mathrm{W}(\tau, \varphi) / W(0,0)$ according to formula (2.17) presented in 2-D plane and 3-D plane, respectively, for the TX-RX distance $d=$ $0.42 \mathrm{~km}$.; other parameters, as above.

Once again, a good agreement between measured data and simulation results is observed. Using the same technique as above, we accept the matching ratio of 0.68 , i.e., $68 \%$. At the same time, there is a significant difference with the results shown in Figs. 5 and 6.

The maximum signal energy is concentrated in the area of buildings located between the RX and TX antennas due to effects of rays arriving at the receiver after diffraction from roofs and corners of buildings, the main features observed during experiments described in [21]-[23]. So, in this location RX2 the street guiding effect is not so obvious as in the previous case. We also note here, that using only the power spectrum in the mutual AOA and TD domains, we cannot properly show two groups of arriving waves, as in Fig. 8, which is characterized by a common azimuth range of $-25^{\circ}$ to $-30^{\circ}$ and different time delays. Each of the two groups describes a different ray travel distance, when all arrivals in the group have the same route. The same situation with effects of reflections of rays from Hotel Torni Tower occurs. Signal power distribution in mutual AOA and TD domains (both in 2-D and 3-D plane) cannot also explain this situation.

We must note, that the proposed formula (2.17) does not describe the sharp fluctuations in the elevation domain. Because

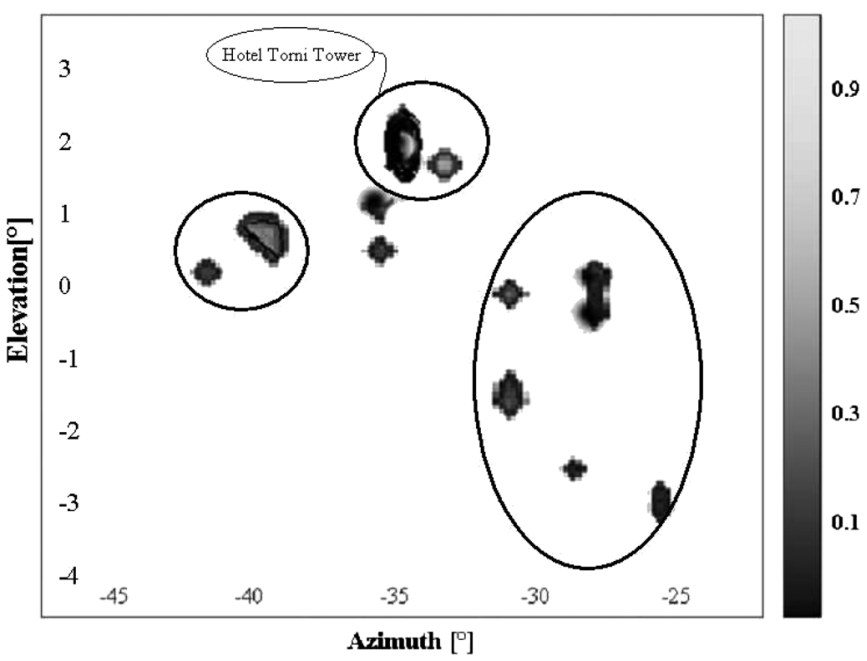

Fig. 12. Measured data in the EOA-AOA plane: antenna is at the rooftop level.

of that, we cannot see matching results for the arrivals from the high tower (Hotel Torni), which is measured in [21], [22] and presented in Fig. 8. Only using signal power spectrum distribution in mutual AOA and EOA domains, the influence of the two groups, clearly seen from measured data (see Fig. 8), will have a satisfactory explanation. Investigating the joint AOA and EOA distribution of total signal power, we can better understand all the effects accounting consolidation of arrivals across the waveguides created street-grid plan.

\section{B. EOA-AOA Signal Power Distribution}

The results of joint EOA-AOA distribution measured in the urban scene shown in Fig. 4, are presented in Fig. 10. It was measured that impinging waves were coming homogeneously over elevation angles of $-4^{\circ}$ to $+2^{\circ}$ with concentrating near the street canyons caused by guiding effect. Fig. 11 shows the results of our simulation of the (a) 2-D and (b) 3-D cases combining (2.18) with (2.21) for the normalized signal power joint AOA-EOA distribution and (2.19) with (2.21) the normalized 

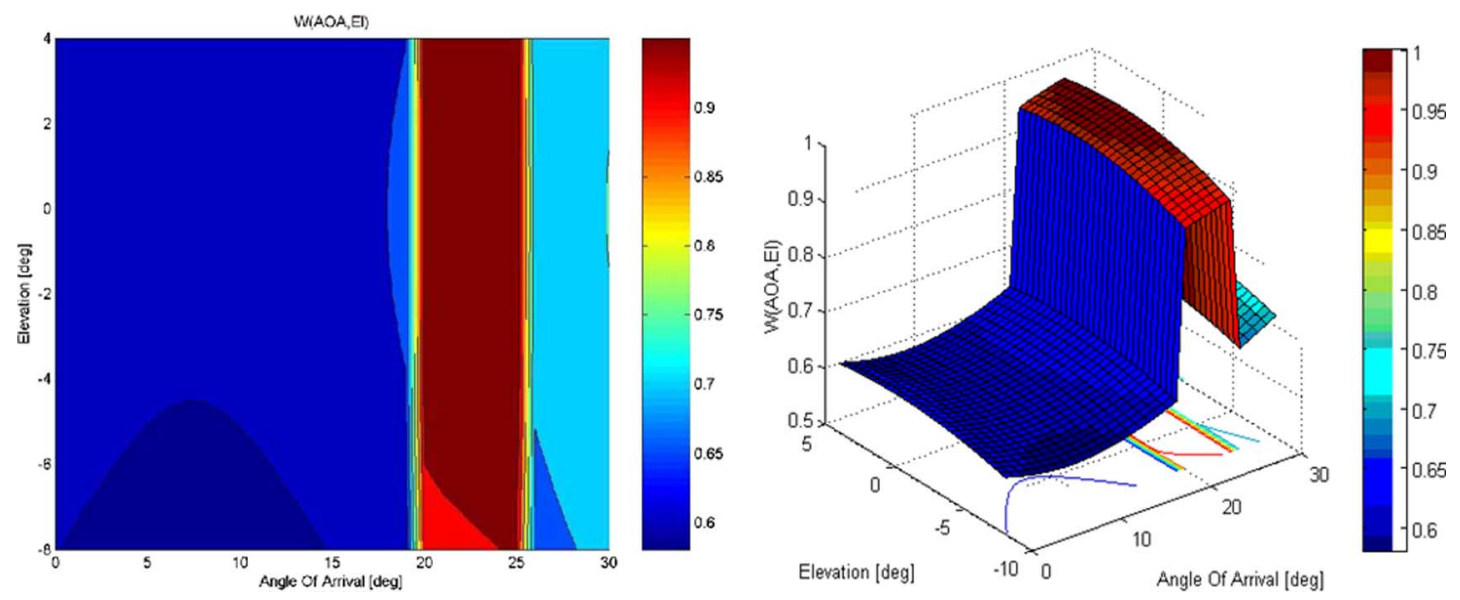

Fig.13. Joint EOA-AOA relative signal power distribution in (a) 2-D and (b) 3-D pictures.
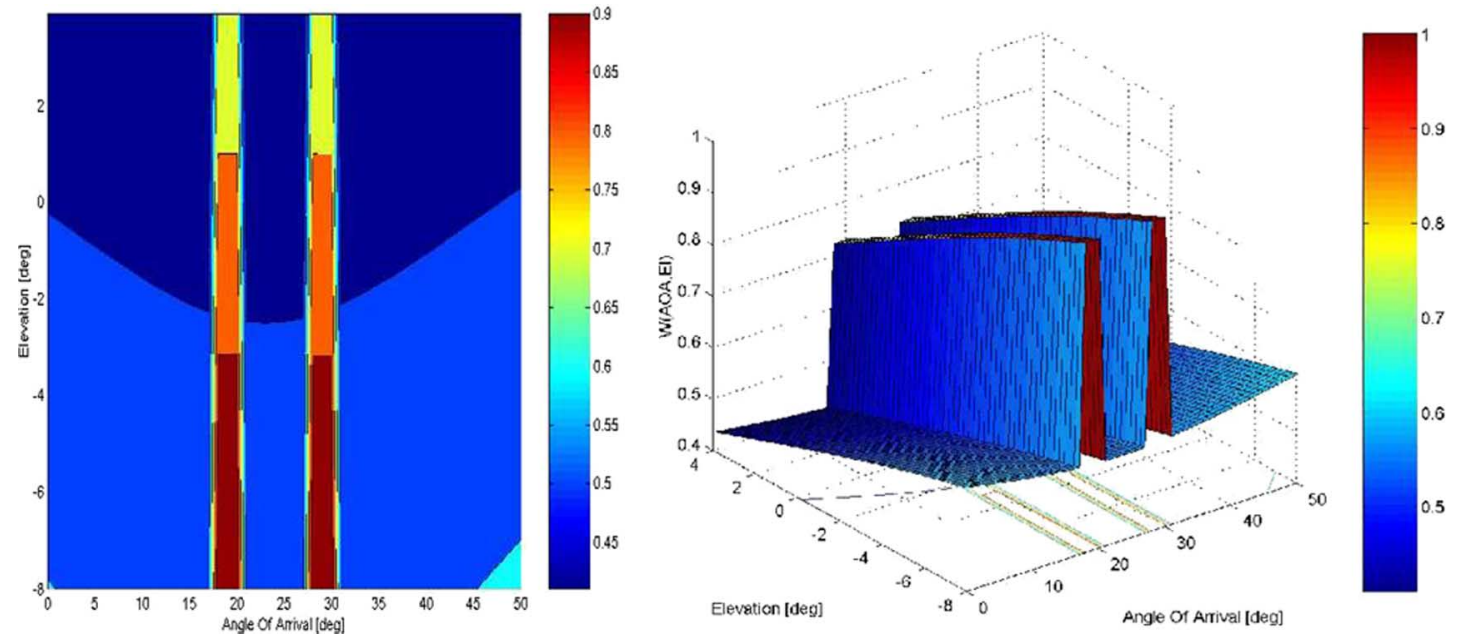

Fig. 14. The relative power spectrum $\widetilde{W}(\theta, \varphi)$ for $\beta=20^{\circ}$ : (a) 2-D and (b) 3-D picture.

signal power joint TD-EOA distribution using the first experimental setup shown in Fig. 4. A good quantitative agreement between measurements and simulation results is also observed in the AOA-EOA joint domain concerning the unique guiding effects of the two streets. The simulation result shows maximum energy of the arriving signal concentrated around elevation angles corresponding to the direction of both streets. In fact, looking now at the mutual EOA-AOA normalized signal power distribution, both in the 2-D plane and the 3-D-plane, presented in Fig. 11 (a) and (b), respectively, and also obtained experimentally (Fig. 10), we obtain the maximum of arriving rays and signal energy concentration around zero elevation angle in the range of $\pm 2^{\circ}$.

For the joint TD-EOA signal power distribution, because the receiver BS antenna was located below the rooftops, the results of measurements in time delay-elevation plane were not so evident. Better results of measurements of joint EOA-AOA and TD-EOA distributions were obtained in the experimental site RX2, where the antenna was located at rooftop level (see Fig. 7). The results of these measurements of joint EOA-AOA signal power distribution are shown in Fig. 12. The corresponding simulation of mutual EOA-AOA distribution for normalized signal power (to signal power along pseudo-LOS direction) is presented in Fig. 13. These results explain the over-rooftop propagation (in our examples RX antenna is at the rooftop level) experimentally observed in [21], [22]. It means that the height of buildings close to the RX define the elevation angles at the RX antenna. In addition, a significant number of wave spread around elevation angle of zero, when there is the guiding effect in the azimuth domain. This effect may be explained by the multiple reflections of rays passing through the street canyon, observed in [21], [22]. Fig. 13 shows that about $80 \%$ of the signal energy arrives at the receiver after multiple reflections, diffractions, and scattering (called Class 2 and Class 3 in [21]-[23]) from the array of buildings on the right-hand side (with respect to TX), which covers an azimuth range of $20^{\circ}-30^{\circ}$ for the whole range of elevation angles. The same effects are followed from the previous results presented in AOA-TD plane [Fig. 9(a) and (b)].

These results are in a good agreement with measurements [21], [22], according to the effects caused by building blocks located right from the TX antenna. The distance between these buildings is about 350-400 meter from RX2, which corresponds to time delay spread of $1.2-1.3 \mu \mathrm{s}$. These buildings are located $25^{\circ}-30^{\circ}$ from the transmitter in the azimuth plane (see Fig. 7). 

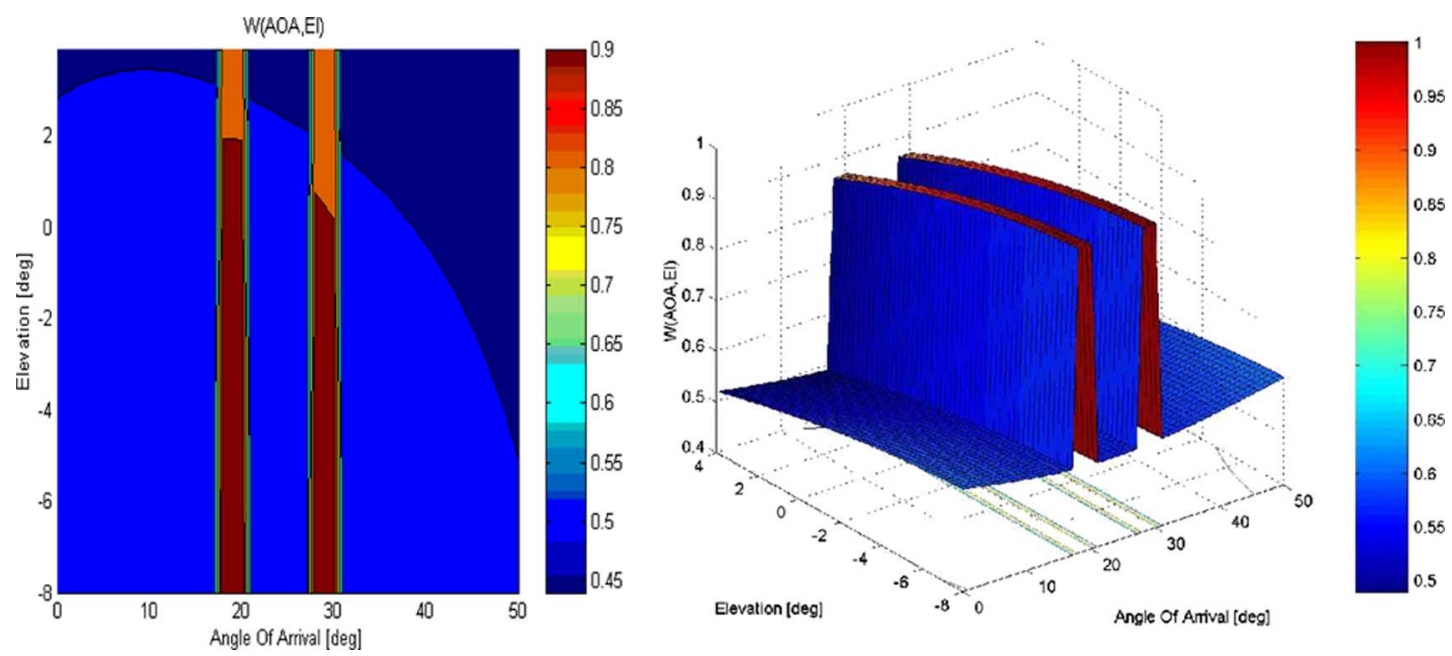

Fig. 15. The relative power spectrum $\widetilde{W}(\theta, \varphi)$ for $\beta=-10^{\circ}$ : (a) 2-D and (b) 3-D picture.
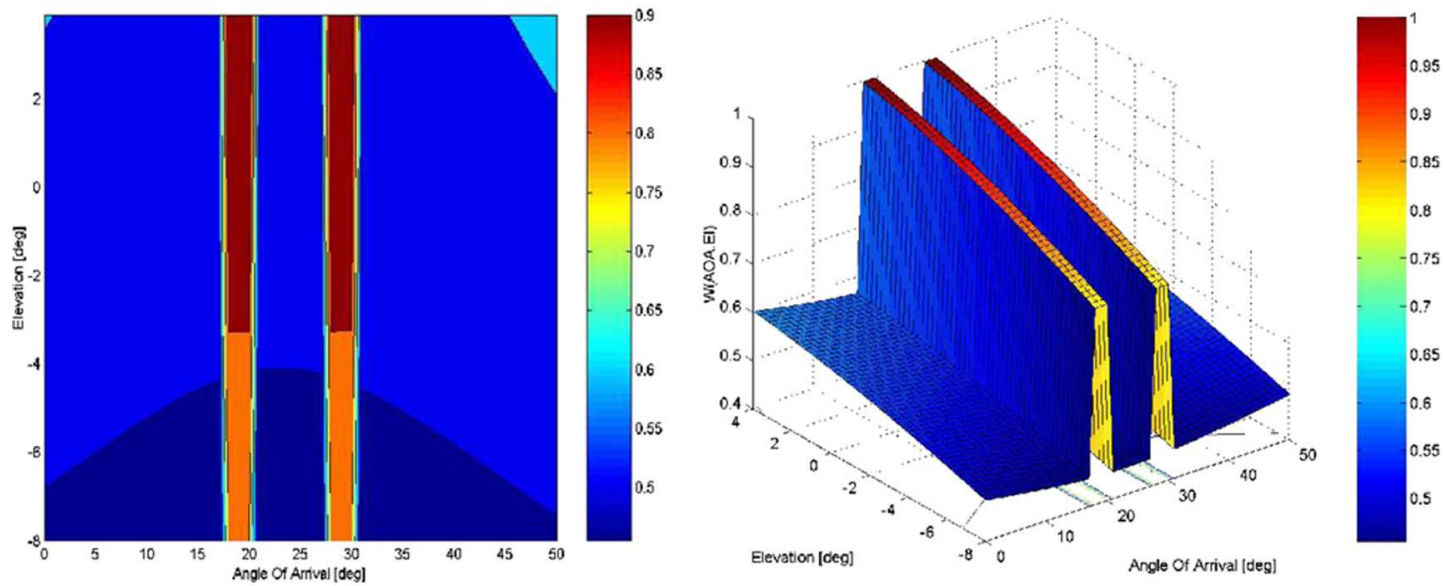

Fig. 16. The relative power spectrum $\widetilde{W}(\theta, \varphi)$ for $\beta=10^{\circ}$ : (a) 2-D and (b) 3-D picture.

\section{NUMERICAL EXPERIMENT WiTH THE VIRTUAL SMART ANTENNA}

The good agreement between theoretical prediction and measured data for different areas of downtown Helsinki [21], [22] allows us to create some virtual numerical experiment with the specific antennas at the base station. In our numerical experiment we will use, for example, the experimental site RX1, shown by Fig. 4, where we will change the BS antenna directivity $\kappa$, azimuth $\varphi$, and tilt $\beta$.

\section{A. Tilt Dependence of the Base Station Antenna}

In this case we will take into account four different tilts of the $\mathrm{RX}$ antenna $\beta=-20^{\circ},-10^{\circ}, 10^{\circ}, 20^{\circ}$. Here again, $\beta>0$ corresponds to tilts above and $\beta<0$ to tilts below the horizon. Figs. 14-17 show these variants, respectively. These changes of the antenna tilt from negative to positive angles in the vertical (elevation) plane reveals that for $\beta>0$ most of the energy arrives from the areas located far from the RX antenna, i.e., caused by multipath components of the total signal due to propagation along the two streets. For $\beta<0$ most energy impinging at the receiving $(\mathrm{RX})$ antenna is located near the direction to the receiver (i.e., around the pseudo-LOS direction) with the channeling effects along both streets. The maximum of wave energy is concentrated at the beginning of the two streets, as it is seen from Fig. 14 (for $\beta=-20^{\circ}$ ) and from Fig. 15 (for $\beta=-10^{\circ}$ ).

At the same time, for $\beta=20^{\circ}$ (Fig. 16) and for $\beta=10^{\circ}$ (Fig. 17), the main signal energy arrives at the receiver from the top-side of both streets (far zone effect).

Both kinds of waves along the two streets are caused by guiding effects of the streets on wave propagation and were defined in [21], [22] as rays of Class 1. In other words, by changing the antenna tilt, we can control the process of serving different users located in zones far and close to the receiver and effects of scatterers located between and/or around the desired users.

\section{B. Azimuth Dependence of the Base Station Antenna}

Now we will turn the array of the antenna in azimuth direction into $\varphi_{0}=5^{\circ}, 50^{\circ}$. These variants are shown in Figs. 18 and 20 for $\varphi_{0}=5^{\circ}$ and $\varphi_{0}=5^{\circ}$, respectively.

Fig. 18 shows the situation when the RX antenna is turned left from the original direction (see Fig. 19), and most energy is received from the azimuth direction of $\varphi_{0}=5^{\circ}$, that is from the street \#1, and less energy is arriving from the street \#2. This result differs from that in the real situation shown by Figs. 5 and $6(\mathrm{a})$, because there are side-rays arriving at the receiver from 

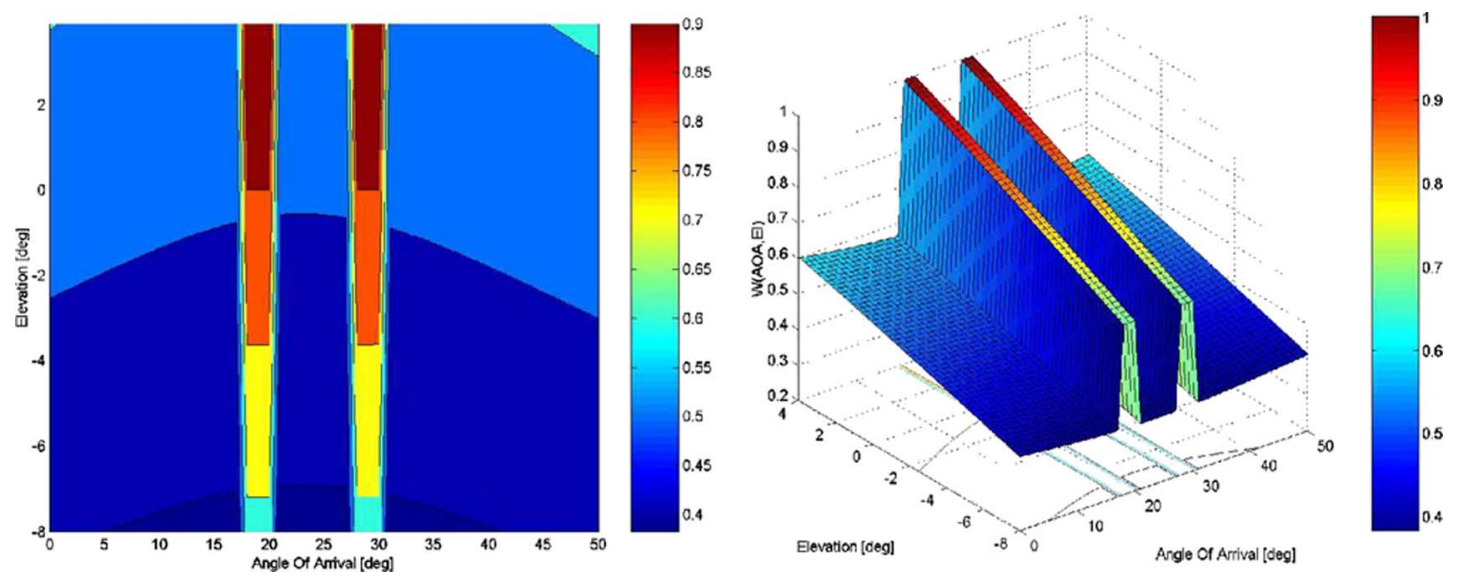

Fig. 17. The relative power spectrum $\widetilde{W}(\theta, \varphi)$ for $\beta=20^{\circ}$ : (a) 2-D and (b) 3-D picture.
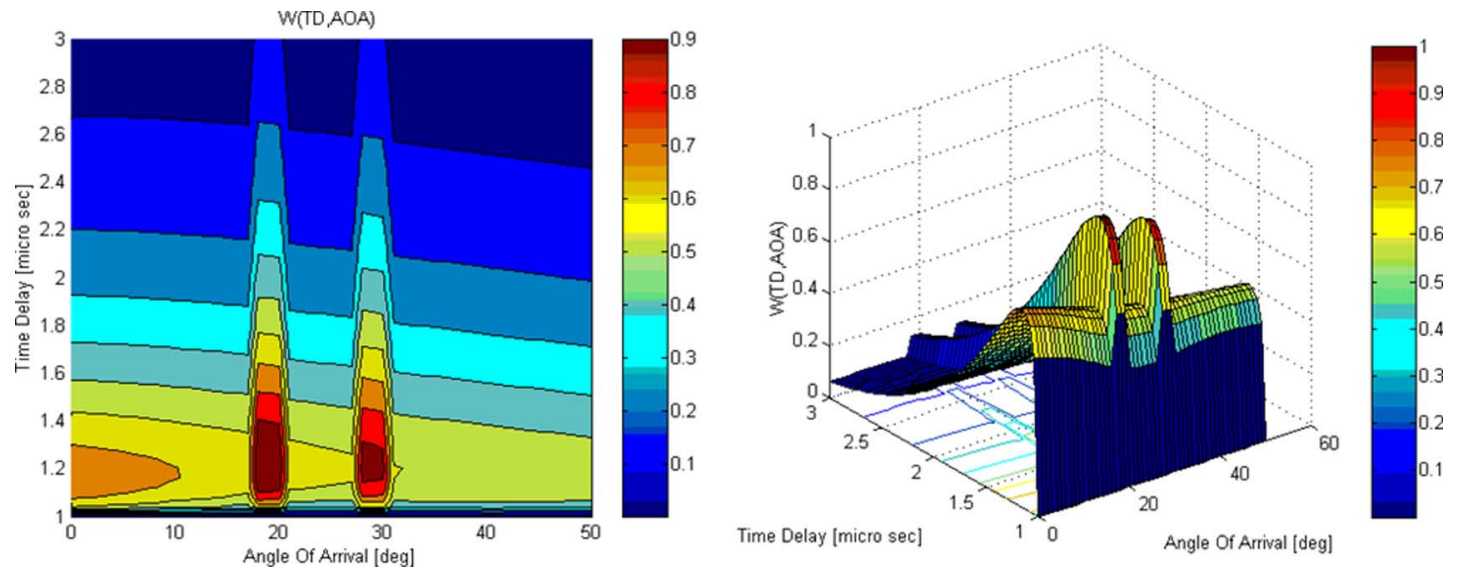

Fig. 18. The (a) 2-D and (b) 3-D normalized power spectrum $\widetilde{W}(\tau, \varphi)$ for $\varphi_{0}=5^{\circ}$.

the left due to reflections from side buildings (called in [21], [22] the rays of Class 2). In the situation described by Fig. 20, the same level of energy is coming from direction $\varphi_{0}=50^{\circ}$ as it is from the streets \#1 and \#2 (see also Fig. 21). In this situation an essential part of the total signal energy is arrived, as shown by Fig. 21, from directions far from the pseudo-LOS located between street \#1 and street \#2. So, the "side" effects—but now from the right side-caused by rays of Class 2 , give the same strong influence on signal energy azimuth distribution as it was observed in the real case described by Figs. 5 and 6(a).

\section{Directivity Dependence of the Base Station Antenna}

Now we will analyze the normalized signal power spectrum $\widetilde{W}(\theta, \varphi)$ for different values of the directivity $\kappa$ of the antenna $(\kappa=10,30)$. Fig. 22 shows these cases, respectively. From this virtual numerical experiment we can understand how the increment of directivity of the RX antenna affects the decrease of the arriving power of the multipath components in the total signal power received by the base station antenna.

It is clear seen that with increase of directivity of the antenna $\kappa$ in the vertical (elevation) plane, most energy arrives at the base station antenna from direction close to elevation angle of zero degree or near this direction, working as a spatial filter to eliminate multipath components arriving from other directions

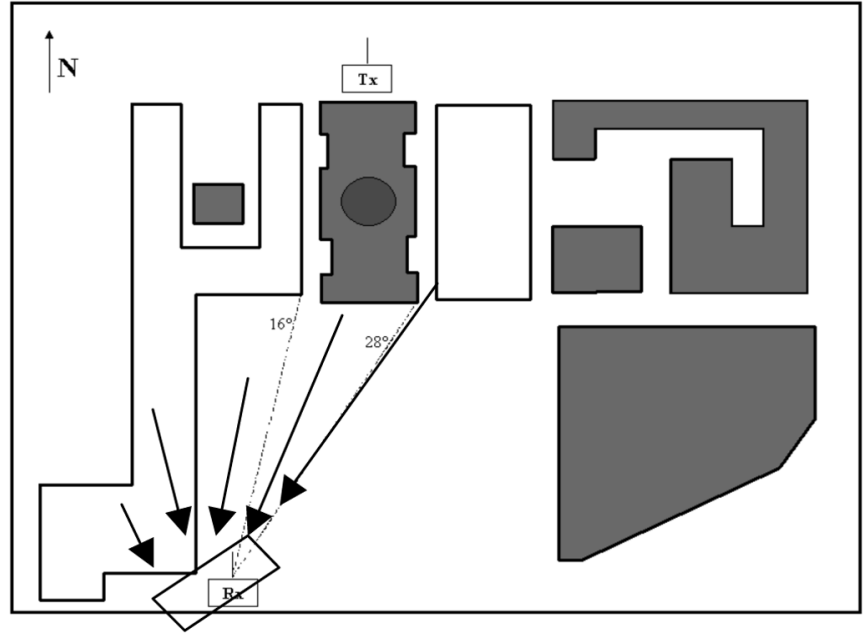

Fig. 19. Detailed map for the situation where the main lobe is directed to $\varphi_{0}=$ $5^{\circ}$.

in the EOA plane. In fact, for $\kappa \leq 10$ all buildings along both streets take part in joint signal power EOA-AOA distribution, whereas for $\kappa>10$ only buildings covered by the main lobe of the antenna with high directivity must be taken into account. 

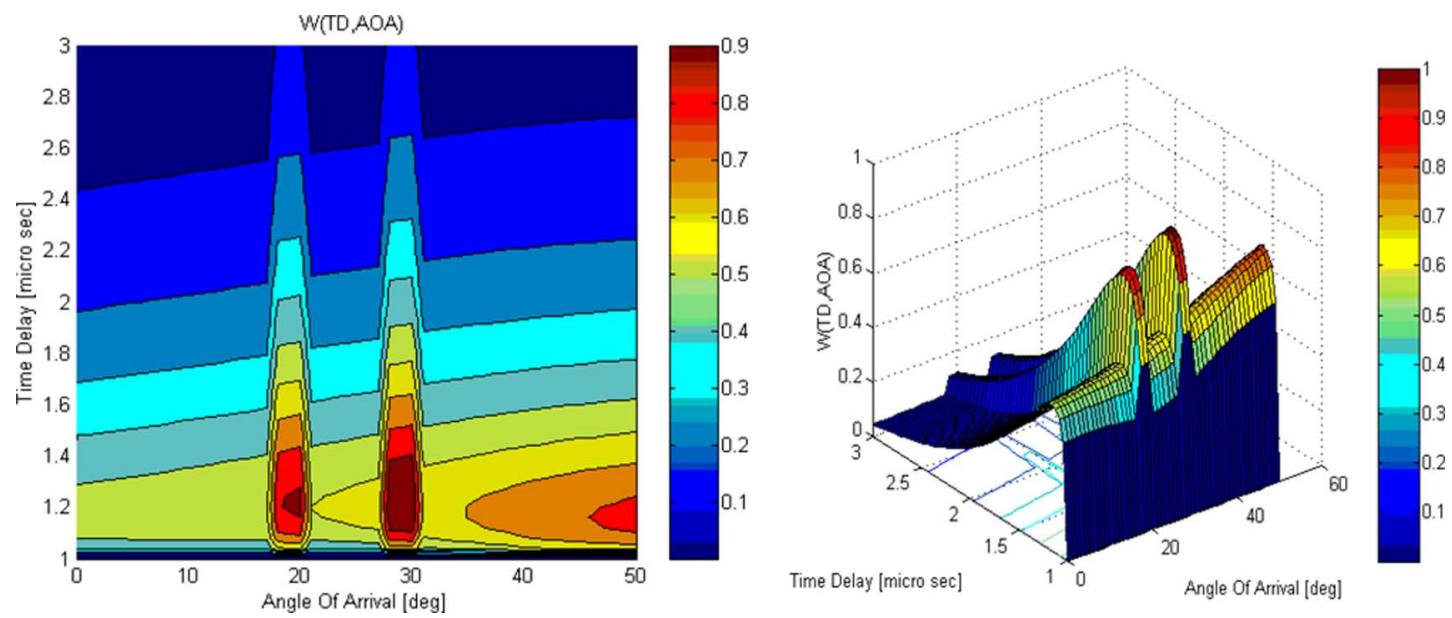

Fig. 20. The (a) 2-D and (b) 3-D normalized power spectrum $\widetilde{W}(\tau, \varphi)$ for $\varphi_{0}=50^{\circ}$.

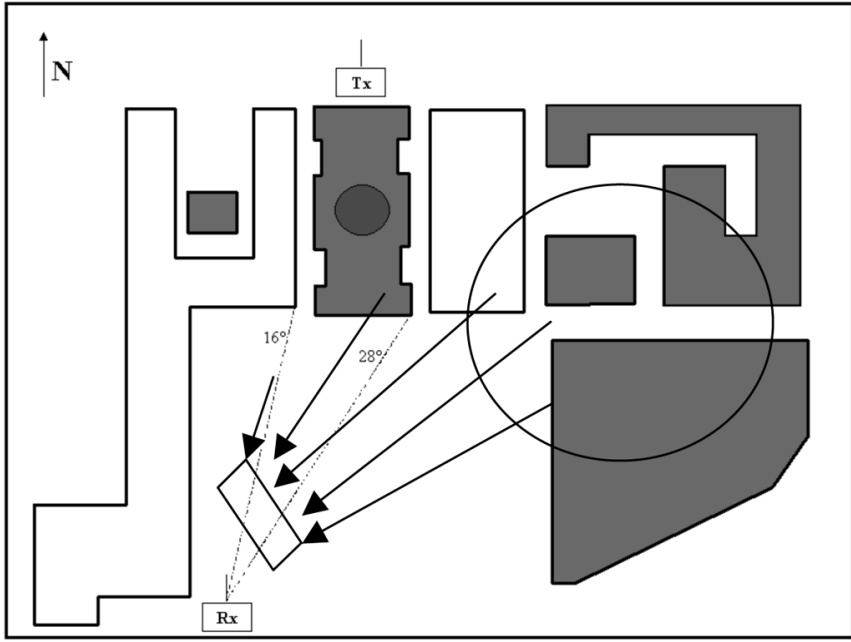

Fig. 21. Detailed map for the situation where the main lobe is directed to $\varphi_{0}=50^{\circ}$.
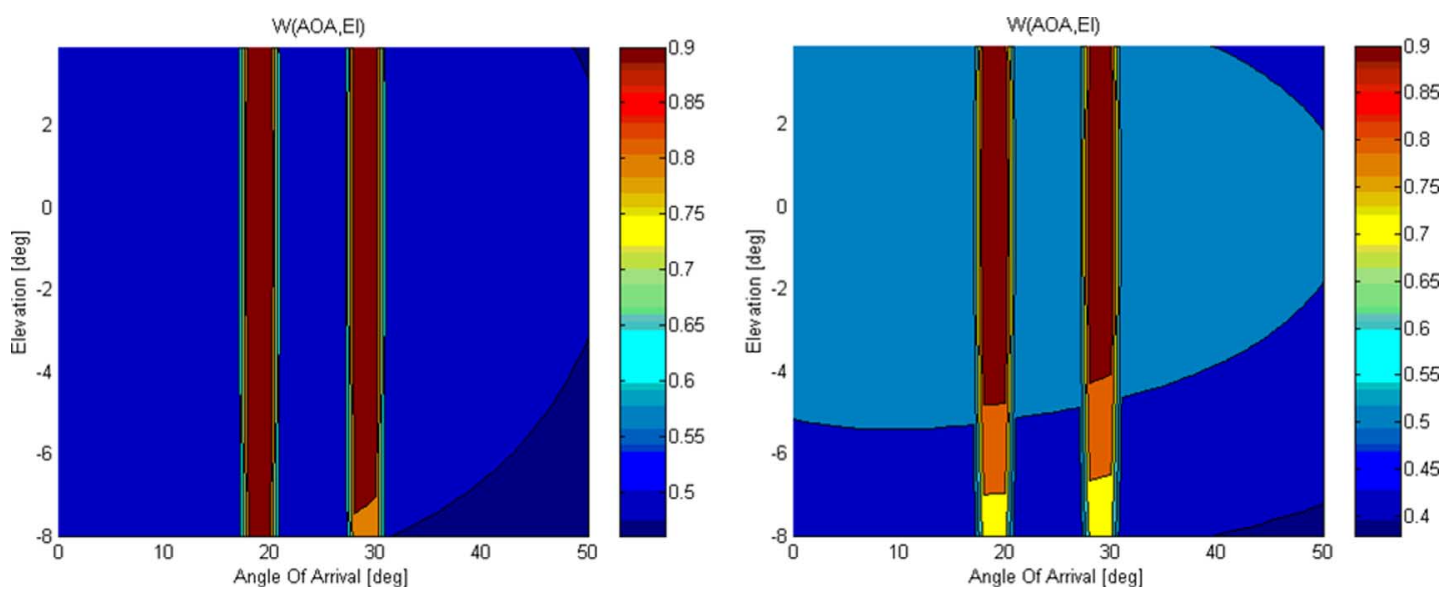

Fig. 22. The normalized signal power spectrum $\widetilde{W}(\theta, \varphi)$ for (a) $\kappa=10$ and (b) $\kappa=30$.

\section{SUMMARY}

In this work, we resolve the problem of mathematical modeling the signal power joint AOA-TD and EOA-AOA distributions in different types of urban areas. We consider, first of all, both arrays of buildings randomly distributed at the terrain and arrays of streets with straight-crossing-grid plan (like Manhattan-grid). The proposed multiparametric model is a general stochastic approach based on combination of statistical description of the terrain features, geometry of obstructions, multislit 
crossing-street waveguide structure (with gaps), and physical description of wave propagation based on Kirchhoff integral equations, considering scattering, reflection and diffraction phenomena.

The proposed approach allows us to eliminate limitations of other models which cannot account for the main features of urban terrain including also such significant factors as antenna heights of TX and RX with respect to building rooftops, RX-TX distance, and crossing-street orientation with respect to the terminal antennas.

Comparing measurement results with the numerical simulation of the proposed model a good agreement was found with experiments described in [21], [22], where it was found experimentally that street canyons carry the strongest power (more than $70 \%$ of all received energy at RX antenna). Furthermore, it was measured that when the RX antenna is on the rooftop level and below, up to $97 \%$ of the total received power belongs to propagation along the streets. Our model confirms these accepted results and gives, in addition, an extremely convenient tool for analyzing the signal power joint AOA, EOA, and TD distributions in the urban scene. Observed effects of virtual numerical experiments carried out in this work, by changing main parameters of the virtual directive antenna assembled at the base station, showed that the proposed novel stochastic approach gives designers of smart antennas a stable planning tool to predict and control a-priori main smart antenna operation parameters. Only topographical properties of the urban terrain and conditions of the terminal antennas with respect to surrounding obstructions are needed for this purpose.

\section{REFERENCES}

[1] B. Widrow, P. E. Mantey, L. J. Griffiths, and B. B. Goode, "Adaptive antenna systems," Proc. IEEE, vol. 55, pp. 2143-2159.

[2] W. F. Gabriel, "Adaptive arrays-an introduction," Proc. IEEE, vol. 64, pp. 239-272, 1976.

[3] J. E. Hadson, Adaptive Array Principles. New York: Peter Peregrinus, 1981.

[4] R. T. Compton, Jr., Adaptive Antennas: Concept and Performance. Englewood Cliffs, NJ: Prentice-Hall, 1988.

[5] W. F. Gabriel, "Adaptive processing array systems," Proc. IEEE, vol. 80, pp. 152-162, 1992.

[6] A. J. Paulraj and C. B. Papadias, "Space-time processing for wireless communication," IEEE Signal Processing Mag., vol. 14, pp. 49-83, 1997.

[7] L. A. L. C. Godara, "Applications of antenna arrays to mobile communications, Part I: performance, improvement, feasibility and system considerations," Proc. IEEE, vol. 85, pp. 1031-1060, 1997.

[8] _ "Applications of antenna arrays to mobile communications, Part II: Beam-forming and direction-of-arrival considerations," Proc. IEEE, vol. 85, pp. 1195-1245, 1997.

[9] L. Chun, G. Stuber, and C. Lea, "Architecture design, frequency planning, and performance analysis for a micro/macro-cell overlaying system," IEEE Trans. Veh. Technol., vol. 46, no. 4, pp. 836-844, 1997.

[10] J. C. Liberti and T. S. Rappaport, Smart Antennas for Wireless Communications. Englewood Cliffs, NJ: Prentice-Hall, 1999.

[11] S. C. Swales, M. Beach, D. Edwards, and J. P. McGeehan, "The performance enhancement of multibeam adaptive base-station antennas for cellular land mobile radio systems," IEEE Trans. Veh. Technol., vol. 39, pp. 56-67, 1990.

[12] P. C. Eggers, J. Toftgard, and A. M. Oprea, "Antenna system for base station diversity in urban small and micro cells," IEEE J. Select. Areas Commun., vol. 11, pp. 1046-1057, 1993.

[13] R. B. Ertel, P. Cardieri, and K. W. Sowerby et al., "Overview of spatial channel models for antenna array communications systems," IEEE Personal Commun., vol. 5, pp. 10-22, 1998.
[14] U. Martin, J. Fuhl, and I. Gaspard et al., "Model scenarios for directionselective adaptive antennas in cellular mobile communication systemsscanning the literature," Wireless Personal Commun. Magazine, vol. 11, pp. 109-129, 1999.

[15] U. Martin, "Spatio-temporal radio channel characteristics in urban macrocells," Proc. Inst. Elect. Eng. Radar, Sonar Navigation, vol. 145, pp. 42-49, 1998.

[16] R. H. Clarke, "A statistical theory of mobile-radio reception," Bell Syst. Tech. J., vol. 47, pp. 957-1000, 1968.

[17] T. Aulin, "A modified model for the fading signal at a mobile radio channel," IEEE Trans. Veh. Technol., vol. 28, pp. 182-203, 1979.

[18] J. Kivinen, T. Korhonen, P. Aikio, R. Gruber, P. Vainikainen, and S. G. Haggman, "Wideband radio channel measurement system at $2 \mathrm{GHz}$," IEEE Trans. Instrum. Meas., vol. 48, pp. 39-44, 1999.

[19] K. Kalliola, H. Laitinen, L. Vaskelainen, and P. Vainikainen, "Realtime 3-D spatial-temporal dual-polarized measurement of wideband radio channel at mobile station," IEEE Trans. Instrum. Meas., vol. 49, pp. 439-448, 2000.

[20] J. Fuhl, J. P. Rossi, and E. Bonek, "High-resolution 3-D direction-ofarrival determination for urban mobile radio," IEEE Trans. Antennas Propag., vol. 45, no. 2, pp. 672-682, 1997.

[21] M. Toeltsch, J. Laurila, K. Kalliola, A. F. Molisch, P. Vainikainen, and E. Bonek, "Statistical characterization of urban spatial radio channels," IEEE J. Select. Areas Commun., vol. 20, pp. 539-549, 2002.

[22] J. Laurila, K. Kalliola, M. Toeltsch, K. Hugl, P. Vainikainen, and E. Bonek, "Wide-band 3-D characterization of mobile radio channels in urban environment," IEEE Trans. Antennas Propag., vol. 50, no. 1, pp. 233-243, 2002.

[23] K. Kalliola, H. Laitinen, P. Vainikainen, M. Toeltsch, J. Laurila, and E. Bonek, "3-D double-directional radio channel characterization for urban macro cellular applications," IEEE Trans. Antennas Propag., vol. 51, no. 12, pp. 3122-3133, 2003.

[24] R. Jenison and K. Fissell, "A comparison of the von Mises and Gaussian basis function for approximating spherical acoustic scatter," IEEE Trans. Neural Networks, vol. 6, no. 5, pp. 1284-1287, 1995.

[25] G. A. Ponomarev, A. N. Kulikov, and E. D. Tel'pukhovskii, Propagation of Ultra-Short Waves in Urban Environments. Tomsk, Rasko: Russia, 1991.

[26] T. S. Rappaport and J. C. Liberti, "A geometrical based model for line-of-sight multipath radio channel," in IEEE Vech. Technology Conf., Atlanta, GA, Apr. 1996, pp. 844-848.

[27] N. Blaunstein, "Wireless communication systems," in Handbook of Engineering Electromagnetics, R. Bansal, Ed. New York: Marcel Dekkel, 2004, ch. 12, pp. 417-481.

[28] H. L. Bertoni, Radio Propagation for Modern Wireless Systems. Englewood Cliffs, NJ: Prentice Hall, 2000.

[29] Q. Spencer et al., "Modeling the statistical time and angle-of-arrival characteristics of an indoor multipath channel," IEEE Trans. Areas Commun., vol. 18, pp. 347-360, 2000.

[30] A. M. Saleh and R. A. Valenzula, "A statistical model for indoor multipath propagation," IEEE J. Select. Areas Commun., vol. SAC-5, pp. $128-137,1987$.

[31] J. B. Andersen and K. I. Pedersen, "Angle-of-arrival statistics for low resolution antennas," IEEE Trans. Antennas Propag., vol. 50, no. 3, pp. 391-395, 2002.

[32] K. I. Pedersen, P. Mogensen, and B. H. Fleury, "A stochastic model of the temporal and azimuthal dispersion seen at the base station in outdoor propagation environments," IEEE Trans. Antennas Propag., vol. 49 , no. 3 , pp. 437-447, 2000 .

[33] _ - "Spatial channel characteristics in outdoor environments and their impact on BS antenna system performance," in IEEE Proc. Veh. Technol. Conf. (VTC'98), Ottawa, Canada, May 1998, pp. 719-724.

[34] _ - "Experimental analysis of the joint statistical properties of azimuth spread, delay spread, and shadow fading," IEEE J. Select. Areas Commun., vol. 20, pp. 523-531, 2002.

[35] C. Cheon, G. Liang, and H. L. Bertoni, "Simulating radio channel statistics for different building environ.," IEEE J. Select. Areas Commun., vol. 19, pp. 1023-1033, 2001.

[36] C. Liang and L. Bertoni, "A new approach to 3-D ray tracing for propagation prediction in cities," IEEE Trans. Antennas Propag., vol. 46, no. 6, pp. 853-863, 1998.

[37] J. B. Andersen et al., "Distribution of phase derivatives in mobile communications," Proc. IEEE, vol. 137, pp. 197-201, 1990.

[38] C. Kloch, G. Liang, J. B. Andersen, G. F. Pedersen, and H. L. Bertoni, "Comparison of measured and predicted time dispersion and direction of arrival for multipath in a small cell environment," IEEE Trans. Antennas Propag., vol. 49, no. 9, pp. 1254-1263, 2001 
[39] A. Abdi, J. Barger, and M. Kaveh, "A parametric model for distribution of the angle of arrival and the associated cor. fun. and power spectrum at the MS," IEEE Trans. Veh. Technol., vol. 51, pp. 425-434, 2002.

[40] N. Blaunstein, "Average field attenuation in the nonregular impedance street waveguide," IEEE Trans. Antennas Propag., vol. 46, no. 6, pp. 1782-1789, 1998.

[41] — , "Prediction of cellular characteristics for various urban environments," IEEE Antennas Propag. Mag., vol. 41, pp. 135-145, 1999.

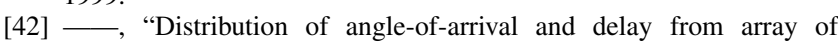
buildings placed on rough terrain for various elevations of base station antenna," J. Commun. Networks, vol. 2, pp. 305-316, 2000.

[43] N. Blaunstein and J. B. Andersen, Multipath Phenomena in Cellular Networks. Norwood, MA: Artech House, 2002.

[44] N. Blaunstein and E. Tsalolihin, "Signal distribution in the azimuth, elevation, and time-delay domains in urban radio communication links," IEEE Antennas Propag. Mag., vol. 46, pp. 171-178, 2004.

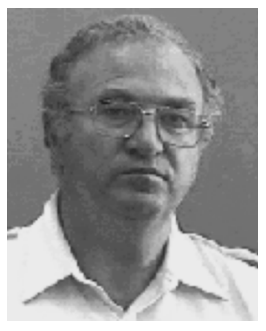

Nathan Blaunstein received the B.Sc. and M.Sc. degrees in radiophysics and electronics from Tomsk University, Tomsk, Russia, in 1972 and 1976, respectively, and the Ph.D., D.Sc., and Professor degrees in radiophysics and electronics from Institute of Geomagnetism, Ionosphere, and Radiowave Propagation (IZMIR), Academy of Science USSR, Moscow, Russia, in 1985 and 1991, respectively.

From 1979 to 1984, he was an Engineer, a Lecturer, and then, from 1984 to 1992, a Senior Scientist, an Associate Professor, and a Professor at Moldavian University, Beltsy, Moldova (former U.S.S.R.). In 1993, he joined the Ben-Gurion University of the Negev, Beer Sheva, Israel, where he was a Senior Scientist in the Department of Electrical and Computer Engineering and then a Visiting Professor in the Wireless Cellular Communication Program, where, since 2001, he has been a Professor in the Department of Communication Systems Engineering. His research interests include problems of radio and optical wave propagation, diffraction and scattering in various media (sub-soil medium, terrestrial environments, troposphere and ionosphere) for purpose of aircraft, mobile-satellite and terrestrial communications, and for optical and radiolocation.

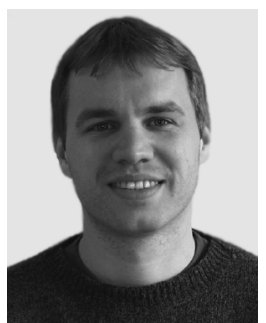

Martin Toeltsch (S'00-M'02) received the Dipl.-Ing. degree (M.S.) in communications engineering and the Dr.Tech. degree from the Technische Universität Wien, Vienna, Austria (TU-Wien), in 1998 and 2002, respectively. In his doctoral thesis, he investigated high-resolution evaluations of directionally resolved radio channel measurements.

From 1992 to 1998, he worked in the field of signal and image processing for medical applications. From 1998 to 2002, he was a member of the Mobile Communications Group at the Institut für Nachrichten-und Hochfrequenztechnik (INTHF), TU-Wien. His research focused on signal processing, OFDM and smart antenna radio channel measurements, as well as propagation and channel modeling. In 2002, he founded SYMENA Software and Consulting company where he develops smart antenna radio network planning and optimization tools.

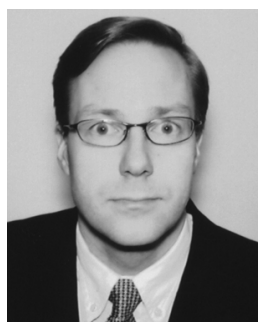

Juha Laurila (S'97-M'00) was born 1970 in Korpilahti, Finland. He received the M.Sc. (E.E.) degree from the Helsinki University of Technology, Finland (Institute of Radio Communications) in 1995 and the Dr.Tech. degree from the Vienna University of Technology, Austria (Institut fuer Nachrichtentechnik und Hochfrequenztechnik) in 2000. In his doctoral thesis he investigated semi-blind algorithms for mobile communications.

From 1995 to 1996, he was with the Helsinki University of Technology and, from 1996 to 2000, with the Vienna University of Technology, working as a Research Engineer at both universities. Since 2000, he has been in various positions with the Nokia Research Center (Radio Technologies Laboratory), Helsinki, Finland. Currently he is acting as a Manager of the Radio Systems competence area. His current interests are related to wireless system research in general. He is also active in the Wireless World Research Forum (WWRF) where he serves as a Vice-Chairman of SIG1 on spectrum aspects. He has authored or coauthored some 30 international reviewed journal and conference publications and one book chapter.

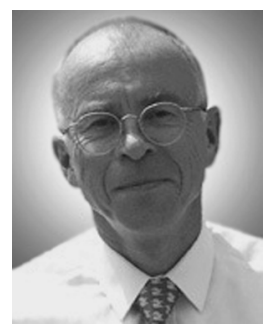

Ernst Bonek (M'73-SM'85) was born in Vienna, Austria, in 1942. He received the Dipl.Ing. and Dr.Techn. degrees (with highest honors) from the Technische Universität Wien (TU Wien).

In 1984, he was appointed Full Professor of Radio Frequency Engineering at the TU Wien. His field of interest is mobile communications at large. Recent contributions concern smart antennas, the characterization of mobile radio channels, and advanced antennas and receiver designs. His group pioneered 3-D superresolution measurements of the urban mobile radio channel, the "double-directional" viewpoint of the mobile radio channel, and propagation-based MIMO channel models. He was a Consultant/Guest Professor at ESA/ESTEC, Noordwijk, The Netherlands, in 1980/81, at TU Lulea, Sweden,in 1997 and with NTTDoCoMo, Yokosuka, Japan, in 2002. He is the initiator of Forschungszentrum Telekommunikation Wien (FTW), a public-private partnership for telecommunications research in Vienna, Austria. Previous fields of research were semiconductors, microwaves, optical communications, and intersatellite links. Altogether, he authored or coauthored some 170 journal and conference publications. He holds several patents on mobile radio technology. He coauthored the book Data Transmission over GSM and UMTS (Berlin, Germany, Springer Verlag) and co-edited Technology Advances of UMTS by (Cachan Cedex,France: Hermes Scientific Publications).

Dr. Bonek was Chairman of URSI Commission C "Signals and Systems" between 1999 and 2002. From 1985 to 1990, he served the IEEE Austria Section as a Chairman. From 1991 to 1994 he was a council member of the Austrian Science Fund, acting as speaker for engineering sciences. From 1996 to 1999, he served on the Board of Directors of the reorganized Post and Telekom Austria. He participated in the European research initiative COST 259 as Chairman of the working group on Antennas and Propagation, and continued to serve in this position in COST 273.

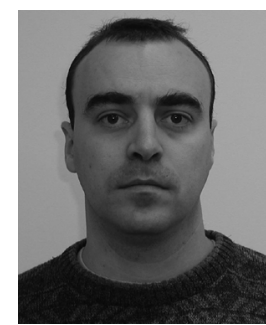

Dmitry Katz was born Moscow. He received the B.Sc. and M.Sc. degrees from the Ben-Gurion University of the Negev (BGU), Beer Sheva, Israel, in 1996 and 2000, respectively, where he is working toward the Ph.D. degree.

He is also a Hardware Team Leader in Albatronics Ltd., Company, Israel. His research interests include aspects of radio propagation in wireless communication links, land and atmospheric, with application to new MIMO systems, WiFi and WiMAX technologies.

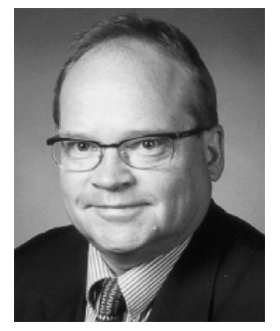

Pertti Vainikainen (M'91) received the degree of Master of Science in Technology, Licentiate of Science in Technology, and Doctor of Science in Technology from Helsinki University of Technology (TKK), Espoo, Finland, in 1982, 1989, and 1991, respectively.

From 1992 to 1993, he was an Acting Professor of radio engineering, from 1993 to 1998, an Associate Professor of radio engineering, and since 1998, a Professor of radio engineering, at the Radio Laboratory, HUT. From 1993 to 1997, he was the Director of the Institute of Radio Communications (IRC), HUT, and in 2000, a Visiting Professor at Aalborg University, Denmark. He is the author or coauthor of six books and over 240 refereed international journal or conference publications and the holder of seven patents. His main fields of interest are antennas and propagation in radio communications and industrial measurement applications of radio waves. 


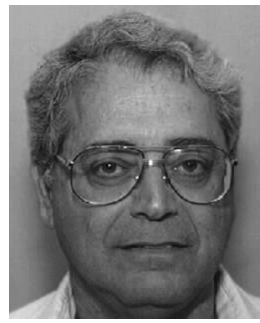

Nissim Tsouri received the B.Sc. and M.Sc. degrees in electrical engineering from the Technion-Israel Institute of Technology, Haifa, Israel, in 1963 and 1968, respectively, and the Ph.D. degree in electrical engineering and computer science from Columbia University, New York, in 1974.

In 1964, he joined the Nuclear Research Center (NRCN), Negev, Israel, where, from 1980 to 1999, he held the position of Manager of the Computer Center. In 1999, he joined the Communication Systems Department at Ben Gurion University of the Negev, Beer Sheva, Israel, shortly after it was founded. Most of his time was devoted to enhance and stabilize the curriculum of the department and to develop the infrastructure of the first and main department's laboratory: the Networks Laboratory. Later on he was in charge of designing and configuring a new laboratory: the High Speed Networks Laboratory. From 2002 to 2005, he was nominated the chairperson of the Communication Systems Department. During that time, three faculty members joined the department and some very important courses were added to the department's curriculum. His research interests are in the areas of QOS in IP networks, IP over ATM networks, traffic management in high speed networks, radiowave propagation and personal and mobile communication systems performance.

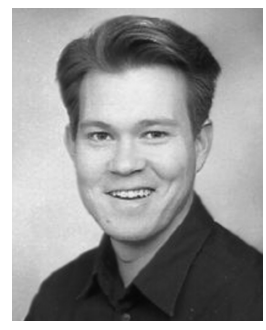

Kimmo Kalliola received the M.Sc., Lic. Sc., and D.Sc. (Tech.) degrees from Helsinki University of Technology (HUT) in 1997, 2000, and 2002, respectively. In his doctoral dissertation, he investigated methods for multidimensional radio channel measurements.

From 1997 to 2001, he was with the Radio Laboratory of HUT, working as a Research Engineer in the field of radio propagation measurements for mobile communications. In 1999, he joined Nokia Research Center (NRC) as a part-time Research Engineer, in
2001, he became a Research Engineer, and since 2004, has been a Research Manager heading the RF Measurements and Applications group at NRC Radio Technologies Laboratory, Helsinki, Finland. His current research interests are related to technologies for flexible spectrum use and radio location tracking. $\mathrm{He}$ is the author or coauthor of several reviewed journal articles and conference papers and has two pending patent applications.

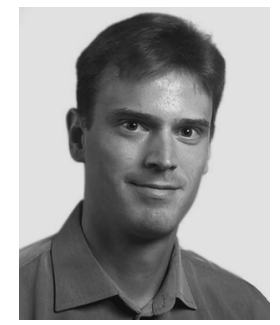

Heikki Laitinen was born in Helsinki, Finland, in 1972. He received the M.Sc. degree in technology from Helsinki University of Technology, Espoo, Helsinki, in 1999, where he is currently working toward the D.Sc. degree

From 1998 to 2004, he was with the Technical Research Centre of Finland (VTT), working as Research Scientist in the fields of radio channel modeling and measurements and radio location methods. Since 2004, he has been working as a GPS Specialist at Space Systems Finland, Espoo. His current work focuses on the development of pseudolite navigation systems. $\mathrm{He}$ is the author or coauthor of more than 10 refereed international journal or conference publications. 